Supporting information for:

\title{
Ship-in-a-Bottle Preparation of Long Wavelength Molecular Antennae in Lanthanide Metal-Organic Frameworks for Biological Imaging
}

\author{
Patrick F. Muldoon ${ }^{1}$, Guillaume Collet ${ }^{2,3}$, Svetlana V. Eliseeva ${ }^{2}$, Tian-Yi Luo ${ }^{1}$, Stéphane Petoud ${ }^{* 1,2}$ and \\ Nathaniel L. Rosi ${ }^{* 1,4}$ \\ 1. Department of Chemistry, University of Pittsburgh, Pittsburgh, PA 15260 \\ 2. Centre de Biophysique Moléculaire, Centre National de la Recherche Scientifique, 45071 Orléans, France \\ 3. Le Studium Loire Valley Institute for Advanced Studies, 1 Rue Dupanloup, 45000 Orléans, France \\ 4. Department of Chemical and Petroleum Engineering, University of Pittsburgh, Pittsburgh, PA 15260
}

\section{Table of Contents:}

1. General Experimental Details

2. Solvothermal MOF Syntheses and Characterization

3. Post Synthetic Modification and Characterization

4. Spectral Data and Imaging 


\section{General Experimental Details}

$\mathrm{Yb}(\mathrm{Cl})_{3} \cdot 6 \mathrm{H}_{2} \mathrm{O}, \mathrm{Yb}\left(\mathrm{O}_{2} \mathrm{CCH}_{3}\right)_{3} \cdot 4 \mathrm{H}_{2} \mathrm{O}$, DMF, 2-aminoterephthalic acid, and acetonitrile were purchased from Sigma-Aldrich and used without further purification. Methyl propiolate was purchased from Matrix Scientific and used without further purification. All DMF used for washing crystals was pre-dried over 4 Å molecular sieves.

${ }^{1} \mathrm{H}$ NMR spectra were obtained using a Bruker Advance $500 \mathrm{MHz}$ spectrometer. Chemical shifts are in parts per million using the residual solvent peak (DMSO-d6, 2.5 ppm) as reference.

X-ray powder diffraction patterns were collected using a Bruker AXS D8 Discover powder diffractometer at $40 \mathrm{kV}, 40 \mathrm{~mA}$ for $\mathrm{Cu} \mathrm{K \alpha},(\lambda=1.5406 \AA)$ with a scan speed of $0.10 \mathrm{sec} / \mathrm{step}$ from 3.0 to $45^{\circ}$ at a step size of $0.02^{\circ}$. The data were analyzed using the EVA program from the Bruker Powder Analysis Software package. The simulated powder patterns were calculated using Materials Studio based on single crystal diffraction data of corresponding MOFs.

Elemental microanalysis of MOFs was performed by the University of Illinois UrbanaChampaign Microanalysis Laboratory with an Exeter Analytical CE440 for CHN analysis.

For both optical microscope images and Microspectrophotometry experiments, crystals in acetonitrile were drop-cast onto borosilicate glass microscope slides. Microspectrophotometry experiments were performed on a CRAIC QDI 2010 microspectrophotometer. The sampling area was fixed at $37 \times 37 \mu \mathrm{m}^{2}$, and the integration time was fixed at $8 \mathrm{~ms}$ for all acquired absorbance spectra. Optical microscopic images were obtained using an Olympus BH-2 microscope.

UV-vis spectra were collected using an Agilent 8453 UV-Vis Spectrometer with a quartz cuvette (10 mm path length) at room temperature.

Measurements of emission, excitation spectra and quantum yields were performed in an integration sphere using samples (solids under DMF) placed into $2.4 \mathrm{~mm}$ inside diameter 
capillaries on the Fluorolog 3 spectrofluorimeter (Horiba Scientific) equipped with a visible photomultiplier tube (PMT) (220-800 nm, R928P; Hamamatsu), and a NIR PMT (950-1650 nm, H10330-75; Hamamatsu). All spectra were corrected for the instrumental functions. Luminescence lifetimes were determined under $355 \mathrm{~nm}$ excitation provided by Nd-YAG laser; emission signal was selected using iHR-320 monochromator, detected using NIR PMT, fed into a $500 \mathrm{MHz}$ bandpass digital oscilloscope (TDS 754C; Tektronix), transferred to a PC where the data were processed with the Origin $8 \circledR$ program. 


\section{Solvothermal MOF Syntheses and Characterization}

Synthesis of MOF-1114(Yb):

Based on the protocol from Luo et. al: ${ }^{1}$ To $3.00 \mathrm{~mL}$ of $0.10 \mathrm{M} \mathrm{YbCl}_{3} \cdot 6 \mathrm{H}_{2} \mathrm{O}$ (DMF) was added $0.250 \mathrm{~mL} \mathrm{H} \mathrm{H}_{2} \mathrm{O}$ followed by $1.5 \mathrm{~mL}$ of $0.05 \mathrm{M}$ 2-aminoterephthalic acid (DMF). The resulting solution was sealed and placed in a preheated $100^{\circ} \mathrm{C}$ oven for 16 hours to yield colorless cubic crystals as product.

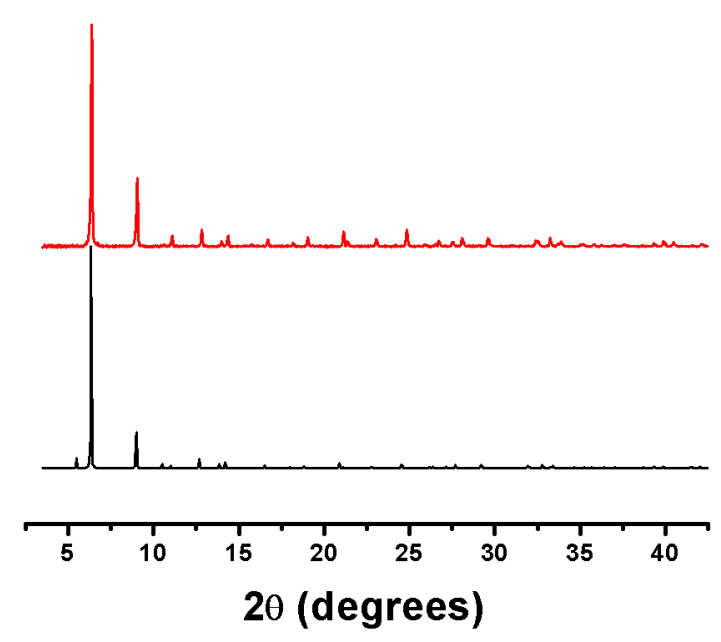

Figure S1: Experimental PXRD pattern for as-synthesized MOF-1114(Yb) washed with DMF (red) and PXRD pattern simulated from single crystal data (black).

Synthesis of MOF-1140(Yb):

$126.7 \mathrm{mg}(0.3 \mathrm{mmol})$ of $\mathrm{Yb}\left(\mathrm{O}_{2} \mathrm{CCH}_{3}\right)_{3} \cdot 4 \mathrm{H}_{2} \mathrm{O}$ was suspended in $3.250 \mathrm{~mL}$ of $12: 1 \mathrm{DMF} / \mathrm{H}_{2} \mathrm{O}$ via sonication for 10 minutes. To the resulting suspension was added $2.75 \mathrm{~mL}$ of $0.05 \mathrm{M} \mathrm{2-}$ aminoterephthalic acid (DMF). The reaction mixture was sonicated for an additional 10 minutes, sealed and placed in a preheated $100^{\circ} \mathrm{C}$ oven for 70 hours to yield colorless plate-shaped crystals. 


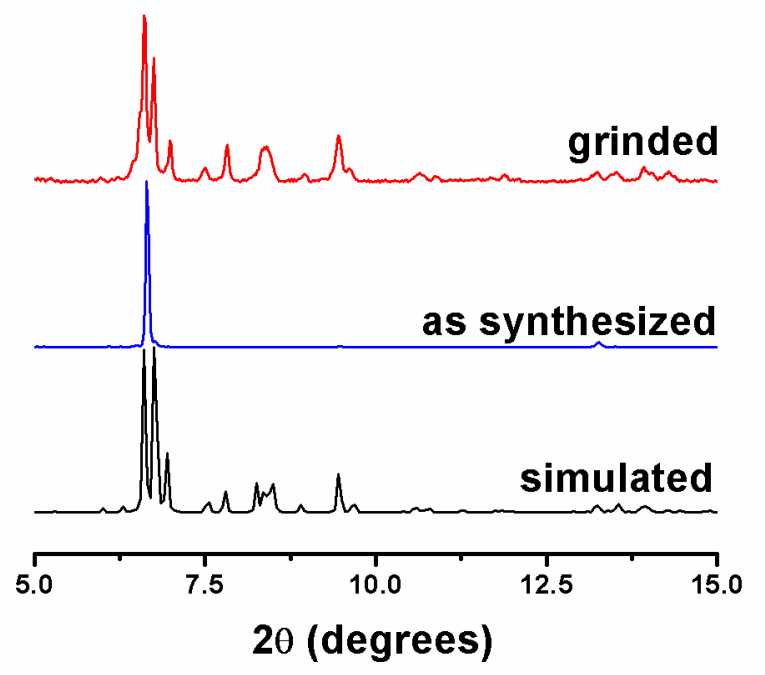

Figure S2: Experimental PXRD pattern for as-synthesized MOF-1140(Yb) washed with DMF exhibiting 001 preferred orientation expected for plate-like crystals (blue), PXRD pattern simulated from single crystal data (black), and PXRD pattern after grinding MOF crystals with mortar and pestle (red).

Table S1: Elemental analysis data for MOF-1140(Yb). Theoretical values calculated based on crystal structure and ${ }^{1} \mathrm{H}$ NMR of dissolved crystals which indicate $9 \%$ of $\mathrm{NH}_{2}$-BDC linkers is replaced with acetates (two acetates per missing linker): $\mathrm{Yb}_{4}\left(\mu_{3}{ }^{-} \mathrm{OH}\right)_{4}\left(\mathrm{NH}_{2-}\right.$ $\mathrm{BDC})_{3.64}\left(\mathrm{CH}_{3} \mathrm{CO}_{2}\right)_{0.72}\left(\mathrm{H}_{2} \mathrm{O}\right)_{6}$

\begin{tabular}{|c|c|c|c|c|c|}
\hline \multirow{2}{*}{ Element } & \multirow{2}{*}{ Theoretical } & \multicolumn{2}{|c|}{ Run 1 } & \multicolumn{2}{c|}{ Run 2 } \\
\cline { 3 - 6 } & & Found & Diff & Found & Diff \\
\hline $\mathrm{C}$ & $23.48 \%$ & $23.34 \%$ & -0.14 & $23.41 \%$ & -0.07 \\
\hline $\mathrm{H}$ & $2.35 \%$ & $1.93 \%$ & -0.42 & $1.88 \%$ & -0.47 \\
\hline $\mathrm{N}$ & $3.26 \%$ & $3.4 \%$ & +0.14 & $3.41 \%$ & +0.15 \\
\hline
\end{tabular}


Single Crystal X-ray Diffraction of MOF-1140(Yb):

Single crystal X-ray diffraction data for MOF-1140(Yb) were collected on a Bruker X8 Prospector Ultra diffractometer equipped with an Apex II CCD detector and an I $\mu$ S micro-focus $\mathrm{CuK} \alpha \mathrm{X}$-ray source $(\lambda=1.54178 \AA)$. Plate-shaped crystal with dimensions $0.08 \times 0.07 \times 0.01$ $\mathrm{mm}^{3}$ was mounted on goniometer. Data was collected under $\mathrm{N}_{2}$ stream at $230 \mathrm{~K}$ and processed using the Bruker APEX II software package.

A monoclinic unit cell (space group $\mathrm{C} 2 / \mathrm{c}$ ) with dimensions $\mathrm{a}=33.4131(14) \AA, \mathrm{b}=52.725(2)$ $\AA, c=26.6616(14) \AA, \alpha=90^{\circ}, \beta=128.378(3)^{\circ}, \gamma=90^{\circ}$, was derived from least squares refinement of 9972 reflections in range of $6.265^{\circ}<2 \theta<116.385^{\circ}$.

Data were collected to $0.83 \AA$ and integrated by Bruker program SAINT, empirical absorption correction was applied using program SADABS. Maximum and minimum transmittance (Tmax, Tmin) values are 0.7519 and 0.4645 , respectively.

Structure was solved using Olex $2^{2}$ with the ShelXT ${ }^{3}$ structure solution program using Direct Methods and refined with the ShelXL ${ }^{4}$ refinement package using Least Squares minimization. Idealized atom positions were calculated for hydrogen atoms (with $\mathrm{d}-(\mathrm{C}-\mathrm{H})=0.940 \AA$ for phenyl rings and $\mathrm{d}-(\mathrm{N}-\mathrm{H})=0.870 \AA$ for amino groups). All the non- $\mathrm{H}$ atoms were refined anisotropically with the exception of O1-O4. All the $\mathrm{H}$ atoms were refined isotropically. DFIX, DANG and FLAT commands were used to constrain locations of $\mathrm{C}$ atoms of phenyl moieties. DELU commands were applied to $\mathrm{C}$ atoms of the same to restrain ADP deviation. ISOR commands were applied to restrain the anisotropy of some $\mathrm{O}$ atoms. Based on the known structure of aminoterephthalate linkers in MOF, SUMP command was used to constrain the total occupancy of disordered $\mathrm{N}$-atoms sharing the same phenyl ring to 1. Crystallographic data are summarized in Table S2. 
Response to checkCIF alert:

PLAT602_ALERT_2_A VERY LARGE Solvent Accessible VOID(S) in Structure

Response: The void(s) in MOF pores should contain molecules of solvent (dimethylformamide).

There are severely disordered solvent molecules that couldn't be located, resulting in the large void space.

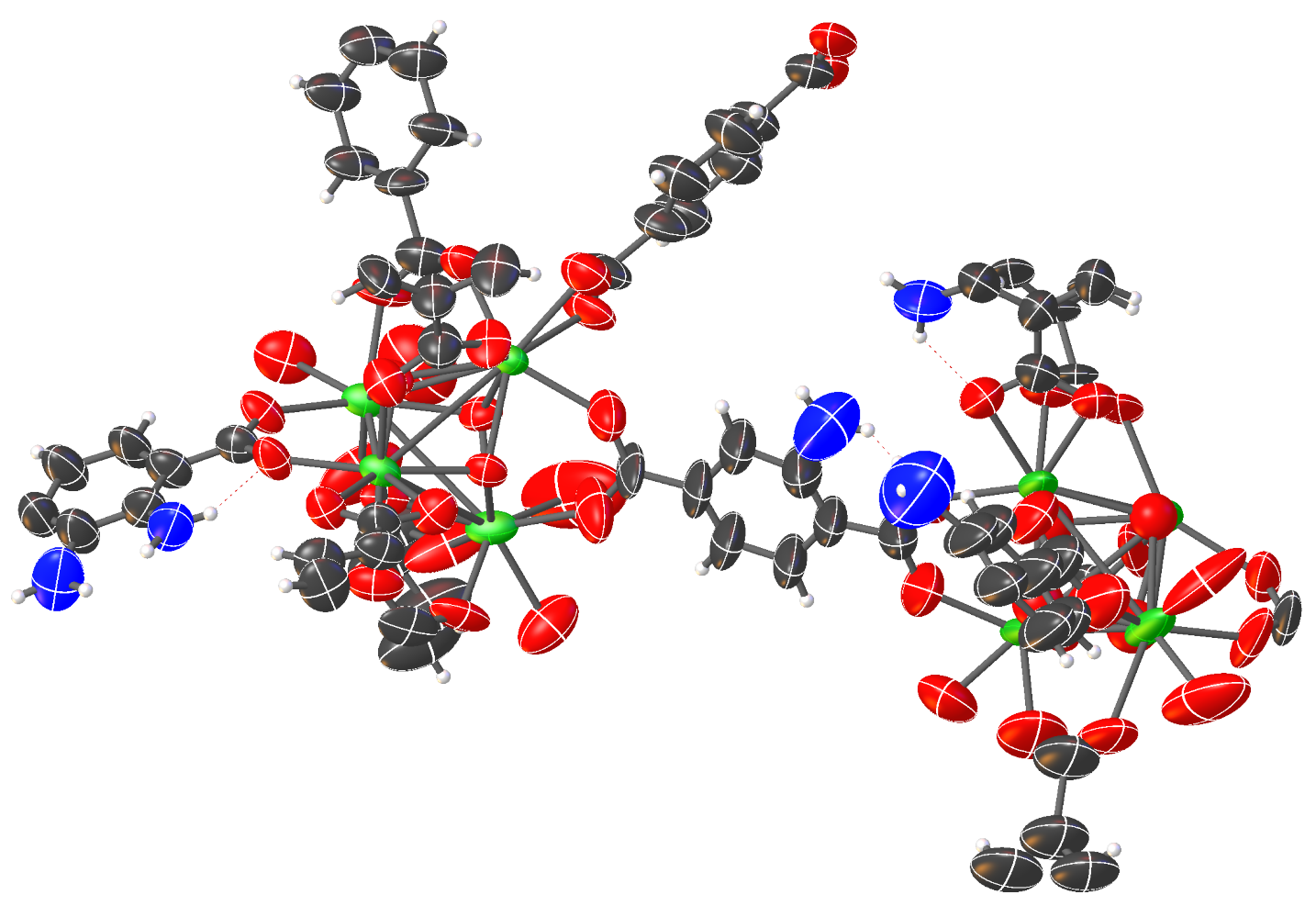

Figure S3: Asymmetric unit of MOF-1140(Yb) produced by $\mathrm{OLEX} 2^{2}$, with all non-H atoms represented by thermal ellipsoids drawn at $50 \%$ probability with the exception of O1-O4 which were refined isotropically ( $\mathrm{O}$, red; $\mathrm{C}$, grey; $\mathrm{N}$, blue; $\mathrm{Yb}^{3+}$, green; $\mathrm{H}$, white spheres). 
Table S2: Crystal data and structure refinement for MOF-1140(Yb)

\begin{tabular}{|c|c|}
\hline Identification code & MOF-1140(Yb) \\
\hline Empirical formula & $\mathrm{C}_{512} \mathrm{H}_{279.97} \mathrm{~N}_{35.98} \mathrm{O}_{397.35} \mathrm{Yb}_{64}$ \\
\hline Formula weight & 24367.55 \\
\hline Temperature/K & 230.0 \\
\hline Crystal system & monoclinic \\
\hline Space group & $\mathrm{C} 2 / \mathrm{c}$ \\
\hline $\mathrm{a} / \AA \AA$ & $33.4131(14)$ \\
\hline $\mathrm{b} / \AA$ & $52.725(2)$ \\
\hline $\mathrm{c} / \AA \AA$ & $26.6616(14)$ \\
\hline$\alpha{ }^{\circ}$ & 90 \\
\hline$\beta /{ }^{\circ}$ & $128.378(3)$ \\
\hline$\gamma /{ }^{\circ}$ & 90 \\
\hline Volume $/ \AA^{3}$ & $36821(3)$ \\
\hline $\mathrm{Z}$ & 1 \\
\hline$\rho_{\text {calc }} / \mathrm{cm}^{3}$ & 1.099 \\
\hline$\mu / \mathrm{mm}^{-1}$ & 7.597 \\
\hline $\mathrm{F}(000)$ & 11263.0 \\
\hline Crystal size $/ \mathrm{mm}^{3}$ & $0.08 \times 0.07 \times 0.01$ \\
\hline Radiation & $\mathrm{CuK} \alpha(\lambda=1.54178)$ \\
\hline $2 \Theta$ range for data collection ${ }^{\circ}$ & 3.352 to 121.124 \\
\hline Index ranges & $-37 \leq \mathrm{h} \leq 37,-59 \leq \mathrm{k} \leq 58,-28 \leq 1 \leq 29$ \\
\hline Reflections collected & 177786 \\
\hline Independent reflections & $26572\left[\mathrm{R}_{\text {int }}=0.2171, \mathrm{R}_{\text {sigma }}=0.1423\right]$ \\
\hline Data/restraints/parameters & $26572 / 625 / 1087$ \\
\hline Goodness-of-fit on $\mathrm{F}^{2}$ & 1.060 \\
\hline Final $R$ indexes $[\mathrm{I}>=2 \sigma(\mathrm{I})]$ & $\mathrm{R}_{1}=0.1535, \mathrm{wR}_{2}=0.3879$ \\
\hline Final $\mathrm{R}$ indexes [all data] & $\mathrm{R}_{1}=0.2418, \mathrm{wR}_{2}=0.4422$ \\
\hline Largest diff. peak/hole / e $\AA^{-3}$ & $2.78 /-1.96$ \\
\hline
\end{tabular}


Table S3: Fractional Atomic Coordinates $\left(\times 10^{4}\right)$ and Equivalent Isotropic Displacement Parameters $\left(\AA^{2} \times 10^{3}\right)$ for MOF-1140(Yb). $U_{\text {eq }}$ is defined as $1 / 3$ of of the trace of the orthogonalised $U_{I J}$ tensor.

\begin{tabular}{|c|c|c|c|c|}
\hline Atom & $x$ & $y$ & $z$ & $\mathrm{U}(\mathbf{e q})$ \\
\hline Yb01 & $6676.3(5)$ & 2495.3(3) & $2383.4(7)$ & $58.0(4)$ \\
\hline Yb02 & $5827.6(6)$ & 2986.3(3) & $1231.1(8)$ & $64.5(5)$ \\
\hline Yb03 & $6136.5(6)$ & $2441.2(4)$ & $674.6(8)$ & $71.1(5)$ \\
\hline Yb04 & $7113.3(6)$ & $2920.4(4)$ & 1694.1(9) & $79.2(6)$ \\
\hline Yb05 & $7025.6(7)$ & $5482.4(4)$ & $1956.8(14)$ & $152.7(16)$ \\
\hline Yb06 & $6790.2(7)$ & 4997.0(4) & $2714.4(17)$ & 176.7(19) \\
\hline Yb07 & $7874.6(9)$ & $5425.0(5)$ & $3699.9(17)$ & $197(2)$ \\
\hline Yb08 & $7913.1(8)$ & $4937.0(5)$ & $2743(2)$ & $226(3)$ \\
\hline O7 & $5968(7)$ & $2552(3)$ & $1368(9)$ & $55(5)$ \\
\hline O8 & 6892(7) & $2512(4)$ & $1711(8)$ & $59(5)$ \\
\hline O6 & 6674(7) & 2899(4) & $2130(8)$ & $60(5)$ \\
\hline $\mathrm{O} 4$ & $7029(10)$ & $5437(5)$ & $2686(13)$ & $97(7)$ \\
\hline $\mathrm{O} 1$ & $7079(10)$ & $5042(5)$ & $1968(13)$ & $101(8)$ \\
\hline O5 & $6241(7)$ & $2878(4)$ & $831(10)$ & $69(6)$ \\
\hline O35 & 6001(9) & 2514(4) & $2400(10)$ & $79(6)$ \\
\hline O18 & $5096(8)$ & $2817(5)$ & $378(11)$ & $91(7)$ \\
\hline $\mathrm{O} 26$ & $6963(8)$ & 2291(4) & $3365(9)$ & $68(6)$ \\
\hline O36 & 5704(9) & $2907(5)$ & $1940(12)$ & $82(6)$ \\
\hline $\mathrm{O} 21$ & $6583(8)$ & 2086(4) & $2113(10)$ & $76(6)$ \\
\hline $\mathrm{O} 25$ & 6989(9) & $2703(5)$ & $3375(10)$ & $83(6)$ \\
\hline $\mathrm{O} 20$ & $7530(9)$ & $2477(5)$ & 2991(11) & $91(7)$ \\
\hline $\mathrm{O} 22$ & $6229(8)$ & 2049(4) & $1094(10)$ & $73(6)$ \\
\hline O11 & $3596(10)$ & $4197(5)$ & $-1751(17)$ & $130(12)$ \\
\hline O34 & $7288(13)$ & $5370(5)$ & $1421(19)$ & $220(20)$ \\
\hline $\mathrm{C} 50$ & $7282(13)$ & 2493(8) & $4372(15)$ & $82(8)$ \\
\hline O10 & $5172(9)$ & $3280(5)$ & $934(13)$ & $105(8)$ \\
\hline $\mathrm{O} 2$ & $7869(12)$ & $5359(6)$ & $2825(15)$ & $128(10)$ \\
\hline O6S & $5806(11)$ & $2134(6)$ & $-156(12)$ & $122(10)$ \\
\hline O14 & $6256(11)$ & $3358(5)$ & $1623(16)$ & $115(9)$ \\
\hline O9 & $5453(10)$ & $3290(5)$ & $330(13)$ & $115(9)$ \\
\hline O12 & $3382(10)$ & $4219(6)$ & $-1105(15)$ & $133(13)$ \\
\hline O39 & $6120(10)$ & 4784(6) & $2644(17)$ & $144(13)$ \\
\hline $\mathrm{C} 26$ & $6660(13)$ & $1692(7)$ & $1752(15)$ & $78(9)$ \\
\hline $\mathrm{O} 40$ & $6143(9)$ & $5210(5)$ & $2725(16)$ & $139(13)$ \\
\hline C35 & $4064(13)$ & 2714(8) & $-567(17)$ & 104(11) \\
\hline
\end{tabular}




\begin{tabular}{|c|c|c|c|c|}
\hline C55 & $5190(14)$ & $2492(8)$ & $2470(20)$ & $114(13)$ \\
\hline C54 & $5345(15)$ & $2700(8)$ & $2370(20)$ & $94(9)$ \\
\hline $\mathrm{C} 25$ & $6473(12)$ & $1952(7)$ & $1652(15)$ & $68(8)$ \\
\hline C53 & $5716(15)$ & $2723(8)$ & $2230(20)$ & $92(9)$ \\
\hline C31 & $6496(14)$ & $1547(7)$ & $1246(18)$ & $106(13)$ \\
\hline C34 & $4462(12)$ & $2577(7)$ & $-497(16)$ & $96(10)$ \\
\hline $\mathrm{C} 42$ & $5453(16)$ & 4997(8) & $2600(20)$ & $122(14)$ \\
\hline O12S & $8424(13)$ & $4654(7)$ & 2633(19) & $181(17)$ \\
\hline $\mathrm{O} 17$ & $5305(8)$ & $2505(5)$ & $-24(11)$ & $102(8)$ \\
\hline $\mathrm{C} 33$ & $5037(15)$ & $2657(9)$ & 27(19) & $99(11)$ \\
\hline C51 & $7370(14)$ & $2264(8)$ & $4688(15)$ & $86(9)$ \\
\hline O8S & $7854(13)$ & $3135(6)$ & $1948(15)$ & $136(12)$ \\
\hline C49 & $7087(14)$ & 2492(8) & $3657(16)$ & $82(9)$ \\
\hline $\mathrm{C} 47$ & $7730(20)$ & $2681(12)$ & $500(30)$ & $186(17)$ \\
\hline $\mathrm{C} 27$ & $6959(14)$ & $1597(7)$ & $2384(17)$ & $91(11)$ \\
\hline C52 & $7550(16)$ & $2282(8)$ & $5308(16)$ & $106(12)$ \\
\hline C38 & $3781(14)$ & $2315(8)$ & $-1412(17)$ & $108(12)$ \\
\hline $\mathrm{O} 33$ & $7864(13)$ & $5066(6)$ & $1909(17)$ & $200(20)$ \\
\hline O19 & $7780(8)$ & $2799(6)$ & $2657(11)$ & 102(9) \\
\hline $\mathrm{O} 38$ & $6203(10)$ & $5316(5)$ & $1198(19)$ & $195(19)$ \\
\hline O16 & $6899(13)$ & $4588(6)$ & $2670(30)$ & 211(19) \\
\hline $\mathrm{O} 23$ & $7958(13)$ & $5810(6)$ & $3444(16)$ & $210(20)$ \\
\hline $\mathrm{O} 37$ & $6079(10)$ & $5030(7)$ & $1690(20)$ & $174(18)$ \\
\hline $\mathrm{O} 27$ & $7258(12)$ & $2750(8)$ & $1052(14)$ & $158(13)$ \\
\hline $\mathrm{C} 24$ & $3618(17)$ & $4128(10)$ & $-1270(30)$ & $117(15)$ \\
\hline O13 & $6981(15)$ & $3309(5)$ & $1820(20)$ & $142(10)$ \\
\hline C57 & $5937(14)$ & $5141(9)$ & $1230(30)$ & $146(18)$ \\
\hline O15 & $7588(16)$ & $4559(7)$ & $2700(30)$ & $280(20)$ \\
\hline O9S & $6860(20)$ & $3237(16)$ & $870(30)$ & $360(50)$ \\
\hline C58 & $5458(12)$ & $5073(8)$ & $580(20)$ & 161(19) \\
\hline $\mathrm{O} 28$ & $6697(16)$ & $2422(9)$ & $508(17)$ & $190(15)$ \\
\hline C5 & $6820(20)$ & $3703(8)$ & $2019(16)$ & $177(17)$ \\
\hline C59 & $5276(13)$ & $5203(8)$ & $30(20)$ & $180(30)$ \\
\hline $\mathrm{C} 29$ & $7013(16)$ & $1199(8)$ & $1990(20)$ & $116(14)$ \\
\hline $\mathrm{C} 41$ & $5928(17)$ & $4996(10)$ & $2600(30)$ & $143(16)$ \\
\hline $\mathrm{C} 30$ & $6655(15)$ & $1298(8)$ & 1339(19) & $114(14)$ \\
\hline C39 & $4319(13)$ & $2387(7)$ & $-903(16)$ & $90(10)$ \\
\hline $\mathrm{C} 17$ & $5150(15)$ & $3348(9)$ & $410(20)$ & $111(11)$ \\
\hline
\end{tabular}




\begin{tabular}{|c|c|c|c|c|}
\hline $\mathrm{C} 4$ & 6492(19) & $3856(9)$ & $2090(20)$ & 190(19) \\
\hline $\mathrm{C} 7$ & $7370(19)$ & $4055(8)$ & $2370(30)$ & 197(19) \\
\hline $\mathrm{C} 40$ & $7867(15)$ & $2601(9)$ & $3034(15)$ & $92(11)$ \\
\hline $\mathrm{C} 45$ & $7110(19)$ & $2557(12)$ & $650(20)$ & $165(13)$ \\
\hline $\mathrm{C} 56$ & $5142(16)$ & $2935(8)$ & $2350(20)$ & 111(11) \\
\hline C6 & $7210(20)$ & $3817(8)$ & $2160(20)$ & $210(20)$ \\
\hline C60 & $5167(13)$ & $4895(8)$ & $620(20)$ & $190(30)$ \\
\hline O13S & $7525(17)$ & $5631(10)$ & $3952(17)$ & $200(18)$ \\
\hline O7S & $5860(20)$ & $2640(11)$ & $-340(20)$ & $230(20)$ \\
\hline $\mathrm{C} 43$ & $5234(15)$ & $5215(8)$ & $2510(30)$ & $140(20)$ \\
\hline C44 & $5236(16)$ & $4775(9)$ & $2510(30)$ & $160(20)$ \\
\hline C32 & $7805(19)$ & $5943(7)$ & $2930(20)$ & $170(20)$ \\
\hline $\mathrm{C} 46$ & $7320(19)$ & $2535(10)$ & $330(20)$ & $194(17)$ \\
\hline $\mathrm{C} 3$ & $6680(20)$ & $4100(10)$ & $2315(19)$ & $210(20)$ \\
\hline $\mathrm{C} 28$ & $7140(15)$ & $1346(8)$ & 2433(19) & $108(13)$ \\
\hline $\mathrm{C} 8$ & $6678(17)$ & $3448(7)$ & $1800(20)$ & $117(11)$ \\
\hline $\mathrm{O} 24$ & $7490(11)$ & $5855(5)$ & $2395(15)$ & $166(17)$ \\
\hline $\mathrm{C} 37$ & $3420(14)$ & $2446(8)$ & $-1469(19)$ & $94(10)$ \\
\hline $\mathrm{C} 2$ & $7065(19)$ & $4208(8)$ & $2451(18)$ & $205(19)$ \\
\hline C36 & $3539(15)$ & 2639(9) & $-1047(18)$ & $110(12)$ \\
\hline $\mathrm{C} 1$ & $7180(20)$ & $4465(10)$ & $2670(30)$ & $220(20)$ \\
\hline $\mathrm{C} 16$ & $7586(19)$ & $5207(10)$ & $1380(20)$ & $200(30)$ \\
\hline C14 & $7171(12)$ & $4744(6)$ & $5250(20)$ & 194(18) \\
\hline $\mathrm{C} 15$ & $7130(13)$ & $4806(7)$ & $4710(17)$ & $210(19)$ \\
\hline $\mathrm{C} 10$ & $7464(15)$ & $4981(7)$ & $4759(17)$ & $210(17)$ \\
\hline C11 & $7839(13)$ & $5093(6)$ & $5350(20)$ & 211(19) \\
\hline C12 & $7881(12)$ & $5031(7)$ & $5882(17)$ & $206(18)$ \\
\hline C13 & $7546(15)$ & $4856(7)$ & $5833(18)$ & $192(17)$ \\
\hline $\mathrm{C} 18$ & $4778(15)$ & $3572(9)$ & $10(20)$ & $116(11)$ \\
\hline $\mathrm{C} 21$ & $4001(17)$ & $3916(9)$ & $-780(30)$ & $125(15)$ \\
\hline $\mathrm{C} 20$ & 4093(18) & $3842(10)$ & $-300(30)$ & $147(17)$ \\
\hline $\mathrm{C} 23$ & $4716(17)$ & $3643(10)$ & $-530(20)$ & $133(14)$ \\
\hline $\mathrm{C} 22$ & $4321(17)$ & $3845(9)$ & $-920(30)$ & $138(16)$ \\
\hline C19 & $4439(19)$ & $3634(11)$ & $110(30)$ & 164(19) \\
\hline $\mathrm{O} 32$ & $7035(13)$ & $4977(6)$ & $3702(19)$ & $165(14)$ \\
\hline C48 & $7970(20)$ & $2649(13)$ & $150(30)$ & $210(20)$ \\
\hline O11S & $8428(15)$ & $5638(12)$ & $4730(20)$ & $260(30)$ \\
\hline O31 & $7688(15)$ & $5224(9)$ & $4310(20)$ & $250(20)$ \\
\hline
\end{tabular}




$\begin{array}{lllll}\text { C9 } & 7390(20) & 5062(11) & 4254(17) & 219(18) \\ \text { C64 } & 9734(17) & 4878(12) & 4424(18) & 240(30) \\ \text { C63 } & 9748(16) & 5175(10) & 5110(30) & 210(30) \\ \text { C61 } & 8880(30) & 5106(18) & 4080(50) & 260(30) \\ \text { O30 } & 8700(16) & 4976(10) & 3520(30) & 290(30) \\ \text { C62 } & 9487(13) & 5061(13) & 4520(20) & 230(30) \\ \text { O29 } & 8712(14) & 5262(9) & 4190(30) & 250(30) \\ \text { N6 } & 7510(30) & 1270(15) & 3100(30) & 150(30) \\ \text { N5 } & 7100(30) & 1698(15) & 2950(40) & 160(40) \\ \text { N3 } & 6340(30) & 4195(15) & 2350(30) & 420(60) \\ \text { N2 } & 6820(30) & 4538(15) & 5140(40) & 380(50) \\ \text { N1 } & 5380(30) & 4559(17) & 2460(50) & 160(30) \\ \text { O3 } & 7690(6) & 4998(5) & 3382(9) & 117(9)\end{array}$


Table S4: Anisotropic Displacement Parameters $\left(\AA^{2} \times 10^{3}\right)$ for MOF-1140(Yb). The Anisotropic displacement factor exponent takes the form: $-2 \pi^{2}\left[\mathrm{~h}^{2} \mathrm{a} * \mathrm{U}_{11}^{2}+2 \mathrm{hka} * \mathrm{~b} * \mathrm{U}_{12}+\ldots\right]$.

\begin{tabular}{|c|c|c|c|c|c|c|}
\hline Atom & $\mathrm{U}_{11}$ & $\mathbf{U}_{22}$ & $\mathbf{U}_{33}$ & $\mathbf{U}_{23}$ & $\mathbf{U}_{13}$ & $\mathrm{U}_{12}$ \\
\hline Yb01 & $46.0(9)$ & $75.0(11)$ & $45.2(8)$ & $10.1(8)$ & $24.4(7)$ & $12.9(7)$ \\
\hline $\mathrm{Yb} 02$ & $42.0(9)$ & $66.5(10)$ & $65.9(10)$ & $16.3(8)$ & $24.0(8)$ & $7.0(7)$ \\
\hline Yb03 & $51.1(10)$ & $101.8(14)$ & $54.3(10)$ & $3.5(9)$ & $29.7(8)$ & $8.0(9)$ \\
\hline Yb04 & $51.4(10)$ & $122.0(16)$ & $64.4(11)$ & $6.7(10)$ & $35.9(9)$ & $-4.7(9)$ \\
\hline Yb05 & $47.9(12)$ & $56.4(12)$ & $157(2)$ & $16.0(13)$ & $-34.2(13)$ & $-1.8(9)$ \\
\hline Yb06 & $37.6(11)$ & $71.4(14)$ & $215(3)$ & $29.3(16)$ & $-23.5(15)$ & $-7.7(9)$ \\
\hline Yb07 & $66.6(15)$ & $112(2)$ & $175(3)$ & $69(2)$ & $-41.9(17)$ & $-39.2(14)$ \\
\hline Yb08 & $35.0(12)$ & $84.4(17)$ & $322(5)$ & $76(2)$ & $-5.4(18)$ & $2.3(10)$ \\
\hline $\mathrm{O} 7$ & $59(11)$ & $60(12)$ & $74(12)$ & $15(10)$ & $56(11)$ & $12(9)$ \\
\hline O8 & $51(11)$ & $103(15)$ & $35(9)$ & $-12(10)$ & $33(9)$ & $6(10)$ \\
\hline O6 & $50(11)$ & $78(14)$ & $42(10)$ & $10(9)$ & $24(9)$ & $8(9)$ \\
\hline O5 & $54(12)$ & $84(15)$ & $72(13)$ & $9(11)$ & $40(11)$ & $-8(10)$ \\
\hline $\mathrm{O} 35$ & $104(16)$ & $83(15)$ & $80(14)$ & $17(12)$ & $72(13)$ & $16(12)$ \\
\hline O18 & $63(14)$ & $102(18)$ & $66(15)$ & $4(13)$ & $20(12)$ & $19(12)$ \\
\hline $\mathrm{O} 26$ & $82(14)$ & $72(14)$ & $45(11)$ & $6(10)$ & $36(11)$ & $14(11)$ \\
\hline O36 & $104(15)$ & $90(16)$ & $121(17)$ & $5(13)$ & $105(15)$ & $-7(12)$ \\
\hline $\mathrm{O} 21$ & $82(14)$ & $67(14)$ & $49(12)$ & $10(10)$ & $26(11)$ & $23(11)$ \\
\hline $\mathrm{O} 25$ & $96(16)$ & $93(16)$ & $63(13)$ & $10(12)$ & $50(12)$ & $-7(13)$ \\
\hline $\mathrm{O} 20$ & $65(15)$ & $140(20)$ & $60(13)$ & $13(14)$ & $37(12)$ & $9(14)$ \\
\hline $\mathrm{O} 22$ & $81(14)$ & $85(15)$ & $64(13)$ & $9(11)$ & $51(12)$ & $32(11)$ \\
\hline O11 & $74(16)$ & $79(18)$ & $140(20)$ & $10(17)$ & $16(18)$ & $-3(13)$ \\
\hline $\mathrm{O} 34$ & $130(20)$ & $57(17)$ & $210(30)$ & $-49(19)$ & $-40(20)$ & $30(16)$ \\
\hline C50 & $69(17)$ & $110(20)$ & $51(13)$ & $-9(13)$ & $29(14)$ & $6(17)$ \\
\hline $\mathrm{O} 10$ & $81(16)$ & $110(19)$ & $99(18)$ & $26(15)$ & $44(15)$ & $23(13)$ \\
\hline O6S & $120(20)$ & $170(30)$ & $74(17)$ & $0(18)$ & $62(17)$ & $10(20)$ \\
\hline $\mathrm{O} 14$ & $120(20)$ & $66(15)$ & $190(20)$ & $21(16)$ & $108(19)$ & $7(14)$ \\
\hline O9 & $66(16)$ & $130(20)$ & 119(19) & $70(16)$ & $41(15)$ & $42(14)$ \\
\hline $\mathrm{O} 12$ & $58(17)$ & $100(20)$ & $120(20)$ & $-1(16)$ & $-4(15)$ & $16(14)$ \\
\hline O39 & $76(17)$ & $86(19)$ & $190(30)$ & $12(19)$ & $43(18)$ & $-12(14)$ \\
\hline $\mathrm{C} 26$ & $71(16)$ & $94(19)$ & $46(14)$ & $10(14)$ & $25(13)$ & $17(15)$ \\
\hline $\mathrm{O} 40$ & $58(15)$ & $88(18)$ & $160(20)$ & $8(18)$ & $14(16)$ & $-5(13)$ \\
\hline $\mathrm{C} 35$ & $51(17)$ & $150(30)$ & $80(20)$ & $-22(19)$ & $26(15)$ & $17(17)$ \\
\hline C55 & $80(20)$ & $80(20)$ & $180(30)$ & $20(20)$ & $90(20)$ & $27(18)$ \\
\hline C54 & $91(18)$ & $90(20)$ & $150(20)$ & $1(19)$ & $97(17)$ & $-2(16)$ \\
\hline $\mathrm{C} 25$ & $65(15)$ & $82(18)$ & $50(15)$ & $14(14)$ & $33(13)$ & $7(13)$ \\
\hline
\end{tabular}




\begin{tabular}{|c|c|c|c|c|c|c|}
\hline C53 & $94(17)$ & $85(19)$ & $130(20)$ & $-3(17)$ & $86(16)$ & $-2(16)$ \\
\hline C31 & $80(20)$ & $90(20)$ & $65(18)$ & $8(17)$ & $2(16)$ & $29(17)$ \\
\hline $\mathrm{C} 34$ & $37(14)$ & $130(20)$ & $105(19)$ & $3(17)$ & $37(14)$ & $-4(15)$ \\
\hline $\mathrm{C} 42$ & $76(19)$ & $70(19)$ & $140(20)$ & $0(20)$ & 31(19) & $-11(16)$ \\
\hline O12S & $100(20)$ & $160(30)$ & $180(40)$ & $30(30)$ & $40(20)$ & $50(20)$ \\
\hline $\mathrm{O} 17$ & $41(12)$ & $160(20)$ & $91(15)$ & $-20(15)$ & $33(12)$ & $30(13)$ \\
\hline C33 & $68(17)$ & $130(20)$ & $78(18)$ & $9(17)$ & $35(15)$ & 11(17) \\
\hline C51 & $100(20)$ & $120(20)$ & $50(13)$ & $-7(13)$ & $50(15)$ & $2(18)$ \\
\hline O8S & $160(30)$ & $160(30)$ & $140(30)$ & $-40(20)$ & $110(20)$ & $-70(20)$ \\
\hline C49 & $84(17)$ & $82(18)$ & $51(12)$ & $2(13)$ & $27(14)$ & $5(16)$ \\
\hline $\mathrm{C} 47$ & $160(30)$ & $320(50)$ & $160(30)$ & $-100(30)$ & $140(30)$ & $-100(30)$ \\
\hline $\mathrm{C} 27$ & $73(19)$ & $90(20)$ & $58(17)$ & $18(16)$ & $12(16)$ & $-2(16)$ \\
\hline C52 & $130(30)$ & $110(30)$ & $48(14)$ & $-14(15)$ & $42(19)$ & $10(20)$ \\
\hline $\mathrm{C} 38$ & $67(18)$ & $140(30)$ & $90(20)$ & $-49(19)$ & $39(16)$ & $13(18)$ \\
\hline $\mathrm{O} 33$ & $120(20)$ & $110(20)$ & $140(30)$ & 23(19) & $-30(20)$ & $30(18)$ \\
\hline O19 & $51(13)$ & $160(20)$ & $54(14)$ & $6(14)$ & 11(11) & $35(14)$ \\
\hline $\mathrm{O} 38$ & $41(15)$ & 84(19) & $230(30)$ & $-20(20)$ & $-32(18)$ & $4(13)$ \\
\hline O16 & $100(20)$ & $67(19)$ & $380(40)$ & $20(20)$ & $100(30)$ & $18(15)$ \\
\hline $\mathrm{O} 23$ & $160(30)$ & $81(18)$ & $140(20)$ & $49(17)$ & $-30(20)$ & $-67(18)$ \\
\hline $\mathrm{O} 37$ & $38(16)$ & $120(20)$ & $210(30)$ & $20(20)$ & $4(19)$ & $18(15)$ \\
\hline $\mathrm{O} 27$ & $120(20)$ & $310(40)$ & $93(18)$ & $-80(20)$ & $95(17)$ & $-110(20)$ \\
\hline $\mathrm{C} 24$ & $54(18)$ & $100(20)$ & $120(20)$ & $10(20)$ & $18(19)$ & 11(17) \\
\hline $\mathrm{O} 13$ & $190(30)$ & $67(17)$ & $240(30)$ & $36(19)$ & $170(20)$ & $14(17)$ \\
\hline $\mathrm{C} 57$ & $19(16)$ & $90(20)$ & $200(30)$ & $0(20)$ & $7(19)$ & $1(15)$ \\
\hline $\mathrm{O} 15$ & $130(30)$ & $80(20)$ & $450(50)$ & $50(30)$ & $100(30)$ & $-21(19)$ \\
\hline O9S & $200(50)$ & $490(110)$ & $230(60)$ & $230(70)$ & $60(40)$ & $-30(60)$ \\
\hline C58 & $26(17)$ & $100(20)$ & $200(30)$ & $-20(20)$ & $-7(19)$ & $-6(15)$ \\
\hline $\mathrm{O} 28$ & $180(30)$ & $300(40)$ & $120(20)$ & $-100(20)$ & $110(20)$ & $-110(30)$ \\
\hline C5 & $150(20)$ & $41(18)$ & $320(30)$ & $10(20)$ & $130(20)$ & $-17(17)$ \\
\hline C59 & $30(20)$ & $110(30)$ & $200(40)$ & $-50(30)$ & $-30(20)$ & $2(18)$ \\
\hline $\mathrm{C} 29$ & $90(20)$ & $80(20)$ & $110(20)$ & $-8(19)$ & $25(19)$ & $18(18)$ \\
\hline $\mathrm{C} 41$ & $69(18)$ & $90(20)$ & $170(20)$ & $10(20)$ & $22(19)$ & $7(17)$ \\
\hline $\mathrm{C} 30$ & $90(20)$ & $100(20)$ & $80(20)$ & $-6(18)$ & $20(18)$ & $33(18)$ \\
\hline C39 & $55(16)$ & $130(20)$ & $90(20)$ & $-23(18)$ & $48(15)$ & $10(16)$ \\
\hline $\mathrm{C} 17$ & $59(17)$ & $130(20)$ & $120(20)$ & $32(19)$ & $47(17)$ & $33(16)$ \\
\hline $\mathrm{C} 4$ & $140(30)$ & $60(20)$ & $350(40)$ & $0(30)$ & $140(30)$ & $-20(20)$ \\
\hline $\mathrm{C} 7$ & $130(30)$ & $50(20)$ & $380(40)$ & $-10(30)$ & $150(30)$ & $-15(19)$ \\
\hline $\mathrm{C} 40$ & $70(20)$ & $150(30)$ & $26(14)$ & $-34(16)$ & $16(15)$ & $10(19)$ \\
\hline
\end{tabular}




\begin{tabular}{|c|c|c|c|c|c|c|}
\hline $\mathrm{C} 45$ & $140(20)$ & $300(40)$ & $110(20)$ & $-90(20)$ & $112(19)$ & $-120(20)$ \\
\hline C56 & $120(20)$ & $110(30)$ & $190(30)$ & $-10(20)$ & $140(20)$ & $-10(20)$ \\
\hline C6 & $150(30)$ & $60(20)$ & $370(40)$ & $-10(30)$ & $140(30)$ & $-30(20)$ \\
\hline C60 & $40(20)$ & $100(30)$ & $210(40)$ & $-20(30)$ & $-40(20)$ & $-5(18)$ \\
\hline O13S & $200(30)$ & $300(40)$ & $130(30)$ & $-110(30)$ & $120(20)$ & $-190(30)$ \\
\hline $\mathrm{O} 7 \mathrm{~S}$ & $280(60)$ & $270(60)$ & $180(40)$ & $50(40)$ & $160(50)$ & $60(50)$ \\
\hline $\mathrm{C} 43$ & $100(30)$ & $70(20)$ & $150(30)$ & $-10(20)$ & $20(30)$ & $-1(19)$ \\
\hline $\mathrm{C} 44$ & $70(20)$ & $80(30)$ & $210(30)$ & $0(30)$ & $30(30)$ & $9(19)$ \\
\hline C32 & $130(30)$ & $39(18)$ & $120(30)$ & $20(18)$ & $-30(20)$ & $-25(18)$ \\
\hline $\mathrm{C} 46$ & $170(30)$ & $320(40)$ & $140(30)$ & $-90(30)$ & $120(20)$ & $-120(30)$ \\
\hline $\mathrm{C} 3$ & $130(30)$ & $70(20)$ & $390(40)$ & $-10(30)$ & $150(30)$ & $0(20)$ \\
\hline $\mathrm{C} 28$ & $90(20)$ & $80(20)$ & $80(20)$ & $-3(18)$ & $16(17)$ & $18(18)$ \\
\hline $\mathrm{C} 8$ & $140(20)$ & $36(16)$ & $230(30)$ & $13(18)$ & $150(20)$ & $-11(15)$ \\
\hline $\mathrm{O} 24$ & $110(20)$ & $56(15)$ & $120(20)$ & $8(15)$ & $-33(17)$ & $-3(14)$ \\
\hline C37 & $72(18)$ & $140(30)$ & $90(20)$ & $-30(19)$ & $62(16)$ & $3(17)$ \\
\hline $\mathrm{C} 2$ & $120(20)$ & $50(19)$ & $400(30)$ & $0(20)$ & $140(30)$ & $-11(17)$ \\
\hline C36 & $70(19)$ & $150(30)$ & $69(19)$ & $1(19)$ & $23(16)$ & $2(19)$ \\
\hline $\mathrm{C} 1$ & $100(20)$ & $70(20)$ & $410(40)$ & $20(30)$ & $120(30)$ & $6(18)$ \\
\hline $\mathrm{C} 16$ & $110(30)$ & $80(20)$ & $180(30)$ & $-20(20)$ & $-30(30)$ & $20(20)$ \\
\hline $\mathrm{C} 14$ & $90(20)$ & $130(30)$ & $260(30)$ & $50(30)$ & $60(20)$ & $-40(19)$ \\
\hline $\mathrm{C} 15$ & $110(20)$ & $150(30)$ & $250(30)$ & $70(30)$ & $50(20)$ & $-36(19)$ \\
\hline $\mathrm{C} 10$ & $110(20)$ & $150(30)$ & $230(30)$ & $80(20)$ & $40(20)$ & $-41(19)$ \\
\hline C11 & $120(30)$ & $140(30)$ & $230(30)$ & $80(30)$ & $30(20)$ & $-30(20)$ \\
\hline C12 & $110(30)$ & $140(30)$ & $230(30)$ & $60(30)$ & $30(30)$ & $-45(19)$ \\
\hline $\mathrm{C} 13$ & $90(20)$ & $130(30)$ & $250(30)$ & $60(30)$ & $50(20)$ & $-14(19)$ \\
\hline C18 & $65(18)$ & $150(20)$ & $140(20)$ & $20(20)$ & $65(18)$ & $29(17)$ \\
\hline $\mathrm{C} 21$ & 71(19) & $120(20)$ & $130(20)$ & $40(20)$ & $30(20)$ & $28(18)$ \\
\hline $\mathrm{C} 20$ & $100(20)$ & $150(30)$ & $130(30)$ & $40(20)$ & $40(20)$ & $60(20)$ \\
\hline $\mathrm{C} 23$ & $90(20)$ & $150(30)$ & $130(30)$ & $40(20)$ & $60(20)$ & $20(20)$ \\
\hline $\mathrm{C} 22$ & $80(20)$ & $130(30)$ & $140(30)$ & $40(20)$ & $40(20)$ & $20(20)$ \\
\hline C19 & $100(20)$ & $160(30)$ & $150(30)$ & $30(30)$ & $40(20)$ & $40(20)$ \\
\hline $\mathrm{O} 32$ & $120(20)$ & $120(20)$ & $200(30)$ & $10(20)$ & $80(20)$ & $-50(19)$ \\
\hline C48 & $180(40)$ & $310(50)$ & $180(40)$ & $-100(40)$ & $120(30)$ & $-100(40)$ \\
\hline O11S & $120(30)$ & $340(70)$ & $160(40)$ & $80(40)$ & $0(30)$ & $-80(40)$ \\
\hline O31 & $150(30)$ & $180(30)$ & $230(30)$ & $100(30)$ & $30(30)$ & $-40(20)$ \\
\hline C9 & $140(30)$ & $150(30)$ & $230(30)$ & $70(20)$ & $40(20)$ & $-50(20)$ \\
\hline C64 & $90(30)$ & $230(50)$ & $190(40)$ & $50(40)$ & $-30(30)$ & $20(30)$ \\
\hline C63 & $70(30)$ & $210(50)$ & $180(40)$ & $10(40)$ & $0(30)$ & $-10(30)$ \\
\hline
\end{tabular}




$\begin{array}{lllllll}\text { C61 } & 90(30) & 190(40) & 230(40) & 60(30) & -30(30) & 0(30) \\ \text { O30 } & 100(30) & 220(40) & 240(40) & 40(30) & -50(30) & 0(30) \\ \text { C62 } & 90(30) & 210(40) & 190(30) & 40(30) & -20(30) & -10(20) \\ \text { O29 } & 60(20) & 170(40) & 260(40) & 80(30) & -30(20) & -20(20) \\ \text { N6 } & 180(60) & 120(50) & 60(40) & 10(30) & 30(40) & -10(40) \\ \text { N5 } & 110(50) & 90(50) & 140(60) & 20(40) & 0(50) & -10(40) \\ \text { N3 } & 210(60) & 190(60) & 510(80) & -10(60) & 60(60) & -70(50) \\ \text { N2 } & 260(60) & 250(60) & 370(70) & 60(60) & 70(60) & -50(50) \\ \text { N1 } & 80(40) & 150(60) & 250(70) & -10(60) & 100(40) & 10(40)\end{array}$


Table S5: Bond Lengths for MOF-1140(Yb).

\begin{tabular}{|c|c|c|c|c|}
\hline Atom Atom & Length/Å & Atom & Atom & Length $/ \AA ̊ \AA$ \\
\hline Yb01 Yb02 & $3.656(2)$ & O9 & $\mathrm{C} 17$ & $1.19(5)$ \\
\hline Yb01 Yb04 & $3.723(2)$ & $\mathrm{O} 12$ & $\mathrm{Yb} 05^{1}$ & $2.38(3)$ \\
\hline Yb01 O7 & $2.252(19)$ & O12 & C24 & $1.21(6)$ \\
\hline Yb01 O8 & $2.311(16)$ & O39 & C41 & $1.26(5)$ \\
\hline Yb01 O6 & $2.234(19)$ & $\mathrm{C} 26$ & $\mathrm{C} 25$ & $1.46(5)$ \\
\hline Yb01 O35 & $2.29(2)$ & $\mathrm{C} 26$ & $\mathrm{C} 31$ & $1.34(5)$ \\
\hline Yb01 O26 & $2.412(19)$ & $\mathrm{C} 26$ & $\mathrm{C} 27$ & $1.41(4)$ \\
\hline Yb01 O21 & $2.24(2)$ & $\mathrm{O} 40$ & C41 & $1.27(5)$ \\
\hline Yb01 O25 & $2.42(2)$ & $\mathrm{C} 35$ & $\mathrm{C} 34$ & $1.42(5)$ \\
\hline Yb01 O20 & $2.24(2)$ & $\mathrm{C} 35$ & $\mathrm{C} 36$ & $1.44(5)$ \\
\hline Yb01 C53 & $3.21(4)$ & C55 & $\mathrm{C} 55^{3}$ & $1.36(8)$ \\
\hline Yb01 C49 & $2.76(3)$ & C55 & C54 & $1.31(5)$ \\
\hline Yb02 Yb03 & $3.664(3)$ & C54 & C53 & $1.50(5)$ \\
\hline Yb02 O7 & $2.318(18)$ & C54 & C56 & $1.40(5)$ \\
\hline Yb02 O6 & $2.353(17)$ & $\mathrm{C} 31$ & $\mathrm{C} 30$ & $1.38(5)$ \\
\hline Yb02 O5 & $2.28(2)$ & C34 & C33 & $1.58(5)$ \\
\hline Yb02 O18 & $2.25(2)$ & C34 & C39 & $1.33(5)$ \\
\hline Yb02 O36 & $2.21(2)$ & $\mathrm{C} 42$ & $\mathrm{C} 41$ & $1.58(7)$ \\
\hline Yb02 O10 & $2.38(3)$ & C42 & $\mathrm{C} 43$ & $1.30(5)$ \\
\hline Yb02 O14 & $2.26(3)$ & $\mathrm{C} 42$ & C44 & $1.32(6)$ \\
\hline Yb02 O9 & $2.48(2)$ & O17 & C33 & $1.27(5)$ \\
\hline Yb02 C33 & $3.12(5)$ & C51 & C52 & $1.37(4)$ \\
\hline $\mathrm{Yb} 02 \mathrm{C} 17$ & $2.72(4)$ & C47 & C46 & $1.38(7)$ \\
\hline Yb03 Yb04 & $3.662(3)$ & $\mathrm{C} 47$ & $\mathrm{C} 48$ & $1.58(8)$ \\
\hline Yb03 O7 & $2.317(17)$ & $\mathrm{C} 27$ & $\mathrm{C} 28$ & $1.43(5)$ \\
\hline Yb03 O8 & $2.342(17)$ & $\mathrm{C} 27$ & N5 & $1.38(10)$ \\
\hline Yb03 O5 & $2.33(2)$ & C52 & $\mathrm{C} 50^{2}$ & $1.37(5)$ \\
\hline $\mathrm{Yb} 03 \mathrm{O} 22$ & $2.28(2)$ & C38 & C39 & $1.48(5)$ \\
\hline Yb03 O6S & $2.39(3)$ & $\mathrm{C} 38$ & C37 & $1.31(5)$ \\
\hline Yb03 O17 & $2.21(2)$ & $\mathrm{O} 33$ & $\mathrm{C} 16$ & $1.34(6)$ \\
\hline Yb03 C33 & $3.14(4)$ & O19 & $\mathrm{C} 40$ & $1.35(5)$ \\
\hline $\mathrm{Yb} 03 \mathrm{O} 28$ & $2.18(4)$ & $\mathrm{O} 38$ & C57 & $1.32(6)$ \\
\hline Yb03 O7S & $2.48(4)$ & O16 & $\mathrm{C} 1$ & $1.13(6)$ \\
\hline Yb04 O8 & $2.29(2)$ & $\mathrm{O} 23$ & $\mathrm{C} 32$ & $1.32(6)$ \\
\hline Yb04 O6 & $2.372(19)$ & $\mathrm{O} 37$ & C57 & $1.16(7)$ \\
\hline Yb04 O5 & $2.348(19)$ & $\mathrm{O} 27$ & $\mathrm{C} 45$ & $1.33(6)$ \\
\hline
\end{tabular}




$\begin{array}{lllll}\text { Yb04 O8S } & 2.41(3) & \mathrm{C} 24 & \mathrm{Yb} 05^{1} & 2.70(5) \\ \text { Yb04 O19 } & 2.21(2) & \mathrm{C} 24 & \mathrm{C} 21 & 1.58(6) \\ \text { Yb04 O27 } & 2.24(3) & \mathrm{O} 13 & \mathrm{C} 8 & 1.22(5) \\ \text { Yb04 O13 } & 2.17(3) & \mathrm{C} 57 & \mathrm{C} 58 & 1.50(6) \\ \text { Yb04 O9S } & 2.45(5) & \mathrm{O} 15 & \mathrm{C} 1 & 1.41(7) \\ \text { Yb05 Yb06 } & 3.636(5) & \mathrm{C} 58 & \mathrm{C} 59 & 1.384(19) \\ \text { Yb05 Yb07 } & 3.656(5) & \mathrm{C} 58 & \mathrm{C} 60 & 1.392(19) \\ \text { Yb05 Yb08 } & 3.706(3) & \mathrm{O} 28 & \mathrm{C} 45 & 1.38(5) \\ \text { Yb05 O4 } & 1.95(3) & \mathrm{C} 5 & \mathrm{C} 4 & 1.46(7) \\ \text { Yb05 O1 } & 2.33(3) & \mathrm{C} 5 & \mathrm{C} 6 & 1.29(7) \\ \text { Yb05 O11 } & 2.46(3) & \mathrm{C} 5 & \mathrm{C} 8 & 1.42(5) \\ \text { Yb05 O34 } & 2.17(6) & \mathrm{C} 59 & \mathrm{C} 60^{1} & 1.50(6) \\ \text { Yb05 O2 } & 2.37(3) & \mathrm{C} 29 & \mathrm{C} 30 & 1.46(6) \\ \text { Yb05 O12 } & 2.38(3) & \mathrm{C} 29 & \mathrm{C} 32^{4} & 1.44(5) \\ \text { Yb05 O38 } & 2.35(2) & \mathrm{C} 29 & \mathrm{C} 28 & 1.25(5) \\ \text { Yb05 C241 } & 2.70(5) & \mathrm{C} 17 & \mathrm{C} 18 & 1.56(6) \\ \text { Yb05 O24 } & 2.32(3) & \mathrm{C} 4 & \mathrm{C} 3 & 1.40(7) \\ \text { Yb06 Yb07 } & 3.647(3) & \mathrm{C} 7 & \mathrm{C} 6 & 1.34(6) \\ \text { Yb06 O4 } & 2.47(3) & \mathrm{C} 7 & \mathrm{C} 2 & 1.42(7) \\ \text { Yb06 O1 } & 2.71(3) & \mathrm{C} 40 & \mathrm{C} 37^{5} & 1.48(5) \\ \text { Yb06 O39 } & 2.41(3) & \mathrm{C} 45 & \mathrm{C} 46 & 1.39(7) \\ \text { Yb06 O40 } & 2.45(3) & \mathrm{C} 56 & \mathrm{C} 56^{3} & 1.57(6) \\ \text { Yb06 O16 } & 2.20(3) & \mathrm{C} 60 & \mathrm{C} 59^{1} & 1.50(6) \\ \text { Yb06 O37 } & 2.25(4) & \mathrm{C} 43 & \mathrm{C} 43^{3} & 1.53(10) \\ \text { Yb06 C41 } & 2.71(6) & \mathrm{C} 44 & \mathrm{C} 44^{3} & 1.54(11) \\ \text { Yb06 C1 } & 3.12(6) & \mathrm{C} 44 & \mathrm{~N} 1 & 1.28(8) \\ \text { Yb06 O32 } & 2.22(4) & \mathrm{C} 32 & \mathrm{C} 29^{6} & 1.44(5) \\ \text { Yb06 O3 } & 2.358(17) & \mathrm{C} 32 & \mathrm{O} 24 & 1.23(5) \\ \text { Yb07 Yb08 } & 3.682(7) & \mathrm{C} 46 & \mathrm{C} 48^{7} & 1.40(8) \\ \text { Yb07 O4 } & 2.41(3) & \mathrm{C} 3 & \mathrm{C} 2 & 1.24(7) \\ \text { Yb07 O2 } & 2.35(3) & \mathrm{C} 3 & \mathrm{~N} 3 & 1.30(10) \\ \text { Yb07 O23 } & 2.22(3) & \mathrm{C} 28 & \mathrm{~N} 6 & 1.46(7) \\ \text { Yb07 O13S } & 2.00(5) & \mathrm{C} 37 & \mathrm{C} 40^{8} & 1.48(5) \\ \text { Yb07 O11S } & 2.43(5) & \mathrm{C} 37 & \mathrm{C} 36 & 1.38(5) \\ \text { Yb07 O31 } & 2.33(5) & \mathrm{C} 2 & \mathrm{C} 1 & 1.43(7) \\ \text { Yb07 O29 } & 2.39(5) & \mathrm{C} 16 & \mathrm{C} 13^{9} & 1.41(2) \\ \text { Yb07 O3 } & 2.35(3) & \mathrm{C} 14 & \mathrm{C} 15 & 1.3900 \\ \text { Yb08 O1 } & 2.28(3) & \mathrm{C} 14 & \mathrm{C} 13 & 1.3900\end{array}$




\begin{tabular}{|c|c|c|c|c|c|}
\hline lb08 & $\mathrm{O} 2$ & $2.25(3)$ & C14 & $\mathrm{N} 2$ & $1.48(8)$ \\
\hline Yb08 & O12S & $2.42(4)$ & C15 & $\mathrm{C} 10$ & 1.3900 \\
\hline b08 & $\mathrm{O} 33$ & $2.23(5)$ & $\mathrm{C} 10$ & C11 & 1.3900 \\
\hline $\mathrm{Yb} 08$ & O15 & $2.24(3)$ & $\mathrm{C} 10$ & C9 & $1.275(19)$ \\
\hline lb08 & C61 & $3.10(10)$ & $\mathrm{C} 11$ & $\mathrm{C} 12$ & 1.3900 \\
\hline Yb08 & $\mathrm{O} 30$ & $2.12(4)$ & $\mathrm{C} 12$ & $\mathrm{C} 13$ & 1.3900 \\
\hline Yb08 & $\mathrm{O} 3$ & $2.268(17)$ & $\mathrm{C} 13$ & $\mathrm{C} 16^{10}$ & $1.41(4)$ \\
\hline 035 & $\mathrm{C} 53$ & $1.34(4)$ & $\mathrm{C} 18$ & $\mathrm{C} 23$ & $1.36(6)$ \\
\hline O18 & $\mathrm{C} 33$ & $1.18(5)$ & $\mathrm{C} 18$ & C19 & $1.36(7)$ \\
\hline $\mathrm{O} 26$ & C49 & $1.22(4)$ & $\mathrm{C} 21$ & $\mathrm{C} 20$ & $1.19(7)$ \\
\hline 36 & C53 & $1.23(4)$ & $\mathrm{C} 21$ & $\mathrm{C} 22$ & $1.38(7)$ \\
\hline $\mathrm{O} 21$ & $\mathrm{C} 25$ & $1.26(4)$ & $\mathrm{C} 20$ & C19 & $1.47(6)$ \\
\hline $\mathrm{O} 25$ & C49 & $1.27(4)$ & $\mathrm{C} 23$ & $\mathrm{C} 22$ & $1.50(6)$ \\
\hline $\mathrm{O} 20$ & $\mathrm{C} 40$ & $1.25(5)$ & $\mathrm{O} 32$ & C9 & $1.266(19)$ \\
\hline $\mathrm{O} 22$ & $\mathrm{C} 25$ & $1.28(3)$ & C48 & $\mathrm{C} 46^{7}$ & $1.40(8)$ \\
\hline O11 & ${\mathrm{Yb} 05^{1}}^{1}$ & $2.46(3)$ & $\mathrm{O} 31$ & C9 & $1.257(19)$ \\
\hline O11 & $\mathrm{C} 24$ & $1.30(6)$ & C64 & $\mathrm{C} 63^{11}$ & $1.394(18)$ \\
\hline O34 & $\mathrm{C} 16$ & $1.37(6)$ & C64 & C62 & $1.387(19)$ \\
\hline $\mathrm{C} 50$ & C51 & $1.39(5)$ & C63 & C64 ${ }^{11}$ & $1.394(18)$ \\
\hline C50 & C49 & $1.59(5)$ & C63 & C62 & $1.384(19)$ \\
\hline $\mathrm{C} 50$ & $\mathrm{C} 52^{2}$ & $1.37(5)$ & C61 & $\mathrm{O} 30$ & $1.39(13)$ \\
\hline $\mathrm{O} 10$ & $\mathrm{C} 17$ & $1.41(5)$ & C61 & C62 & $1.62(9)$ \\
\hline $\mathrm{O} 14$ & $\mathrm{C} 8$ & $1.27(4)$ & C61 & O29 & $1.13(13)$ \\
\hline
\end{tabular}

Symmetry transformations used to generate equivalent atoms:

${ }^{1} 1-\mathrm{X}, 1-\mathrm{Y},-\mathrm{Z} ;{ }^{2} 3 / 2-\mathrm{X}, 1 / 2-\mathrm{Y}, 1-\mathrm{Z} ;{ }^{3} 1-\mathrm{X},+\mathrm{Y}, 1 / 2-\mathrm{Z} ;{ }^{4} 3 / 2-\mathrm{X},-1 / 2+\mathrm{Y}, 1 / 2-\mathrm{Z} ;{ }^{5} 1 / 2+\mathrm{X}, 1 / 2-\mathrm{Y}, 1 / 2+\mathrm{Z} ;{ }^{6} 3 / 2-\mathrm{X}, 1 / 2+\mathrm{Y}, 1 / 2-\mathrm{Z}$; ${ }^{7} 3 / 2-\mathrm{X}, 1 / 2-\mathrm{Y},-\mathrm{Z} ;{ }^{8}-1 / 2+\mathrm{X}, 1 / 2-\mathrm{Y},-1 / 2+\mathrm{Z} ;{ }^{9}+\mathrm{X}, 1-\mathrm{Y},-1 / 2+\mathrm{Z} ;{ }^{10}+\mathrm{X}, 1-\mathrm{Y}, 1 / 2+\mathrm{Z} ;{ }^{11} 2-\mathrm{X}, 1-\mathrm{Y}, 1-\mathrm{Z}$ 
Table S6: Bond Angles for MOF-1140(Yb).

\begin{tabular}{|c|c|c|c|c|c|}
\hline Atom & Atom Atom & Angle ${ }^{\circ}$ & Atom & Atom Atom & Angle $/^{\circ}$ \\
\hline Yb02 & Yb01 Yb04 & $.75(5)$ & O37 & Yb06 C1 & $100.0(14)$ \\
\hline $\mathrm{O} 7$ & Yb01 Yb02 & $37.5(5)$ & $\mathrm{O} 37$ & Yb06 O3 & $143.8(14)$ \\
\hline $\mathrm{O} 7$ & Yb01 Yb04 & $76.6(4)$ & C41 & Yb06 Yb05 & $121.5(11)$ \\
\hline O7 & Yb01 O8 & $70.3(6)$ & C41 & Yb06 Yb07 & $126.5(12)$ \\
\hline O7 & Yb01 O35 & $72.8(7)$ & C41 & Yb06 O1 & $139.4(14)$ \\
\hline $\mathrm{O} 7$ & Yb01 O26 & $141.0(7)$ & C41 & Yb06 C1 & $115.7(15)$ \\
\hline $\mathrm{O} 7$ & Yb01 O25 & $132.3(7)$ & $\mathrm{C} 1$ & Yb06 Yb05 & $111.9(12)$ \\
\hline $\mathrm{O} 7$ & Yb01 C53 & $64.9(9)$ & $\mathrm{C} 1$ & Yb06 Yb07 & $109.4(10)$ \\
\hline $\mathrm{O} 7$ & Yb01 C49 & $146.0(9)$ & $\mathrm{O} 32$ & Yb06 Yb05 & $130.1(8)$ \\
\hline $\mathrm{O} 8$ & Yb01 Yb02 & $76.9(5)$ & $\mathrm{O} 32$ & Yb06 Yb07 & $72.8(7)$ \\
\hline $\mathrm{O} 8$ & Yb01 Yb04 & $35.7(5)$ & $\mathrm{O} 32$ & Yb06 O4 & $101.1(10)$ \\
\hline $\mathrm{O} 8$ & Yb01 O26 & $138.9(7)$ & $\mathrm{O} 32$ & Yb06 O1 & $146.9(11)$ \\
\hline O8 & Yb01 O25 & $134.4(7)$ & $\mathrm{O} 32$ & Yb06 O39 & $73.2(12)$ \\
\hline $\mathrm{O} 8$ & Yb01 C53 & $131.0(9)$ & $\mathrm{O} 32$ & Yb06 O40 & $71.7(13)$ \\
\hline O8 & Yb01 C49 & $142.9(9)$ & $\mathrm{O} 32$ & Yb06 O37 & $140.7(16)$ \\
\hline O6 & Yb01 Yb02 & $38.3(4)$ & $\mathrm{O} 32$ & Yb06 C41 & $73.4(16)$ \\
\hline O6 & Yb01 Yb04 & $37.3(5)$ & $\mathrm{O} 32$ & Yb06 C1 & $98.5(15)$ \\
\hline O6 & Yb01 O7 & $72.9(6)$ & $\mathrm{O} 32$ & Yb06 O3 & $75.3(10)$ \\
\hline O6 & Yb01 O8 & $70.9(7)$ & $\mathrm{O} 3$ & Yb06 Yb05 & $78.7(6)$ \\
\hline O6 & Yb01 O35 & 98.4(7) & $\mathrm{O} 3$ & Yb06 Yb07 & $39.1(7)$ \\
\hline O6 & Yb01 O26 & $132.8(7)$ & $\mathrm{O} 3$ & Yb06 O4 & $74.7(9)$ \\
\hline O6 & Yb01 O21 & $148.1(7)$ & $\mathrm{O} 3$ & Yb06 O1 & 71.7(7) \\
\hline O6 & Yb01 O25 & $79.5(7)$ & $\mathrm{O} 3$ & Yb06 O39 & $138.5(10)$ \\
\hline O6 & Yb01 O20 & $91.4(9)$ & $\mathrm{O} 3$ & Yb06 O40 & $135.3(9)$ \\
\hline O6 & Yb01 C53 & $77.9(9)$ & $\mathrm{O} 3$ & Yb06 C41 & $148.7(14)$ \\
\hline O6 & Yb01 C49 & $106.6(10)$ & $\mathrm{O} 3$ & Yb06 C1 & $70.5(12)$ \\
\hline $\mathrm{O} 35$ & Yb01 Yb02 & $74.1(5)$ & $\mathrm{Yb} 05$ & Yb07 Yb08 & $60.67(10)$ \\
\hline $\mathrm{O} 35$ & Yb01 Yb04 & $132.6(5)$ & Yb06 & Yb07 Yb05 & $59.71(8)$ \\
\hline $\mathrm{O} 35$ & Yb01 O8 & $143.1(7)$ & Yb06 & $\mathrm{Yb} 07 \mathrm{Yb} 08$ & $60.98(10)$ \\
\hline $\mathrm{O} 35$ & Yb01 O26 & 74.3(7) & $\mathrm{O} 4$ & Yb07 Yb05 & $29.3(6)$ \\
\hline $\mathrm{O} 35$ & Yb01 O25 & $73.8(8)$ & $\mathrm{O} 4$ & Yb07 Yb06 & $42.3(6)$ \\
\hline $\mathrm{O} 35$ & Yb01 C53 & $20.6(9)$ & $\mathrm{O} 4$ & Yb07 Yb08 & $76.2(6)$ \\
\hline $\mathrm{O} 35$ & Yb01 C49 & $73.7(10)$ & $\mathrm{O} 4$ & Yb07 O11S & $142.6(16)$ \\
\hline $\mathrm{O} 26$ & Yb01 Yb02 & $143.8(5)$ & $\mathrm{O} 2$ & Yb07 Yb05 & $39.4(8)$ \\
\hline $\mathrm{O} 26$ & Yb01 Yb04 & $142.3(5)$ & $\mathrm{O} 2$ & Yb07 Yb06 & $78.5(8)$ \\
\hline $\mathrm{O} 26$ & Yb01 O25 & $53.5(7)$ & $\mathrm{O} 2$ & Yb07 Yb08 & $35.8(8)$ \\
\hline
\end{tabular}




\begin{tabular}{|c|c|c|c|c|c|}
\hline 26 & Yb01 C53 & $89.8(10)$ & $\mathrm{O} 2$ & Yb07 O4 & $66.9(10)$ \\
\hline 26 & Yb01 C49 & $26.3(9)$ & $\mathrm{O} 2$ & Yb07 O11S & $136.2(15)$ \\
\hline & Yb01 Yb02 & $122.2(5)$ & $\mathrm{O} 2$ & Yb07 O29 & $77(2)$ \\
\hline 21 & Yb01 Yb04 & $116.2(6)$ & $\mathrm{O} 2$ & Yb07 O3 & $70.1(9)$ \\
\hline & Yb01 O7 & $84.8(7)$ & $\mathrm{O} 23$ & Yb07 Yb05 & $71.3(9)$ \\
\hline & Yb01 O8 & $80.5(8)$ & $\mathrm{O} 23$ & Yb07 Yb06 & $127.1(7)$ \\
\hline & Yb01 O35 & $96.1(9)$ & $\mathrm{O} 23$ & Yb07 Yb08 & $111.2(14)$ \\
\hline & Yb01 O26 & $78.5(7)$ & $\mathrm{O} 23$ & Yb07 O4 & $85.1(10)$ \\
\hline 21 & Yb01 O25 & $132.0(8)$ & $\mathrm{O} 23$ & Yb07 O2 & $75.9(16)$ \\
\hline 021 & Yb01 O20 & $92.9(9)$ & $\mathrm{O} 23$ & Yb07 O11S & $76.7(15)$ \\
\hline 21 & Yb01 C53 & $113.1(9)$ & $\mathrm{O} 23$ & Yb07 O31 & $141(2)$ \\
\hline 21 & Yb01 C49 & $104.7(10)$ & $\mathrm{O} 23$ & Yb07 O29 & $99.9(16)$ \\
\hline $\mathrm{O} 25$ & Yb01 Yb02 & $100.5(6)$ & $\mathrm{O} 23$ & Yb07 O3 & $145.4(14)$ \\
\hline 25 & Yb01 Yb04 & $103.4(5)$ & O13S & Yb07 Yb05 & $103.2(12)$ \\
\hline 25 & Yb01 C53 & $71.8(10)$ & O13S & Yb07 Yb06 & $93.0(8)$ \\
\hline 25 & Yb01 C49 & $27.3(9)$ & O13s & Yb08 & $153.5(8)$ \\
\hline $\mathrm{O} 20$ & Yb01 Yb02 & $127.8(7)$ & O13S & Yb07 O4 & $80.8(12)$ \\
\hline j20 & Yb01 Yb04 & $70.7(6)$ & O13S & $\mathrm{Yb} 07 \mathrm{O} 2$ & $140.5(16)$ \\
\hline 20 & Yb01 O7 & $142.2(7)$ & $\mathrm{O} 13$ & Yb07 O23 & $79.4(19)$ \\
\hline 20 & Yb01 O8 & $72.1(7)$ & O13S & Yb07 O11S & $64.0(18)$ \\
\hline $\mathrm{O} 20$ & Yb01 O35 & $144.7(8)$ & O13 & Yb07 O31 & $62.9(19)$ \\
\hline 20 & Yb01 O26 & $74.2(8)$ & O13S & Yb07 O29 & $138(2)$ \\
\hline $\mathrm{D} 20$ & Yb01 O25 & $74.8(8)$ & O13S & Yb07 O3 & $124.5(10)$ \\
\hline 20 & Yb01 C53 & $146.3(10)$ & O11s & Yb07 Yb05 & $147.4(12)$ \\
\hline $\mathrm{O} 20$ & Yb01 C49 & $71.0(10)$ & O11s & Yb07 Yb06 & $145.0(12)$ \\
\hline 553 & Yb01 Yb02 & $55.6(8)$ & O11S & Yb07 Yb08 & $140.9(15)$ \\
\hline 553 & Yb01 Yb04 & $112.3(8)$ & $\mathrm{O} 31$ & Yb07 Yb05 & $126.4(10)$ \\
\hline C49 & Yb01 Yb02 & $124.9(8)$ & $\mathrm{O} 31$ & Yb07 Yb06 & $69.2(11)$ \\
\hline C49 & Yb01 Yb04 & $124.3(8)$ & $\mathrm{O} 31$ & Yb07 Yb08 & $107.9(15)$ \\
\hline $\mathrm{C} 49$ & Yb01 C53 & $81.5(11)$ & $\mathrm{O} 31$ & Yb07 O4 & $99.2(11)$ \\
\hline Yb01 & Yb02 Yb03 & $61.21(4)$ & $\mathrm{O} 31$ & Yb07 O2 & $141.9(16)$ \\
\hline O7 & Yb02 Yb01 & $36.3(5)$ & $\mathrm{O} 31$ & Yb07 O11S & $76.5(16)$ \\
\hline $\mathrm{O} 7$ & $\mathrm{Yb02} \mathrm{Yb03}$ & $37.8(4)$ & $\mathrm{O} 31$ & Yb07 O29 & $100.5(19)$ \\
\hline $\mathrm{O} 7$ & Yb02 O6 & $69.5(7)$ & $\mathrm{O} 31$ & Yb07 O3 & $72.1(15)$ \\
\hline $\mathrm{O} 7$ & Yb02 O10 & $138.4(8)$ & $\mathrm{O} 29$ & Yb07 Yb05 & $116.6(18)$ \\
\hline $\mathrm{O} 7$ & Yb02 O9 & $136.0(9)$ & $\mathrm{O} 29$ & Yb07 Yb06 & $118.2(12)$ \\
\hline O7 & Yb02 C33 & $64.7(9)$ & O29 & Yb07 Yb08 & $66.3(16)$ \\
\hline O7 & Yb02 C17 & $143.5(12)$ & $\mathrm{O} 29$ & Yb07 O4 & $141.4(19)$ \\
\hline
\end{tabular}




\begin{tabular}{|c|c|c|c|c|c|}
\hline 6 & Yb02 Yb01 & $36.1(5)$ & O29 & Yb07 O11S & $75(2)$ \\
\hline & Yb02 Yb03 & $78.3(5)$ & $\mathrm{O} 3$ & Yb07 Yb05 & $78.3(5)$ \\
\hline 6 & Yb02 O10 & $137.9(8)$ & $\mathrm{O} 3$ & Yb07 Yb06 & $39.3(4)$ \\
\hline 6 & Yb02 O9 & $132.5(8)$ & $\mathrm{O} 3$ & Yb07 Yb08 & $36.3(5)$ \\
\hline 6 & Yb02 C33 & 130.7 & $\mathrm{O} 3$ & Yb07 O4 & $76.0(8)$ \\
\hline 6 & Yb02 C17 & $146.0(11)$ & $\mathrm{O} 3$ & Yb07 O11S & $134.1(14)$ \\
\hline $\mathrm{O} 5$ & Yb02 Yb01 & $80.6(5)$ & $\mathrm{O} 3$ & Yb07 O29 & 79.0( \\
\hline O5 & $\mathrm{Yb} 02 \quad \mathrm{Yb} 03$ & $37.8(5)$ & Yb05 & Yb08 Yb06 & 58.6 \\
\hline 5 & Yb02 O7 & $72.8(6)$ & Yb07 & Yb08 Yb05 & 59.3 \\
\hline $\mathrm{O} 5$ & Yb02 O6 & $74.5(7)$ & Yb07 & Yb08 Yb06 & 59.0 \\
\hline $\mathrm{O} 5$ & $\mathrm{Yb} 02 \mathrm{O} 10$ & 134.9 & $\mathrm{O} 1$ & Yb08 Yb05 & $36.9(7)$ \\
\hline $\mathrm{O} 5$ & Yb02 O9 & 78.3( & $\mathrm{O} 1$ & Yb08 Yb06 & 46.4 \\
\hline $\mathrm{O} 5$ & Yb02 C33 & $75.6(9)$ & $\mathrm{O} 1$ & Yb08 Yb07 & $84.8(7)$ \\
\hline O5 & Yb02 C17 & $103.8(12)$ & $\mathrm{O} 1$ & Yb08 O12S & $126.8(11)$ \\
\hline $\mathrm{O} 18$ & Yb02 Yb01 & $111.0(6)$ & $\mathrm{O} 1$ & Yb08 C61 & $142(2)$ \\
\hline 18 & Yb02 Yb03 & 71.4(7) & $\mathrm{O} 2$ & Yb08 Yb05 & $37.8(8)$ \\
\hline 18 & Yb02 O7 & $75.7(8)$ & $\mathrm{O} 2$ & Yb08 Yb06 & $77.9(8)$ \\
\hline 18 & Yb02 O6 & $144.9(8)$ & $\mathrm{O} 2$ & Yb08 Yb07 & $37.6(8$ \\
\hline 18 & Yb02 O5 & 91.3(9) & $\mathrm{O} 2$ & Yb08 O1 & 74.4( \\
\hline 18 & Yb02 O10 & 73.9(9) & $\mathrm{O} 2$ & Yb08 O12S & 135. \\
\hline 18 & $\mathrm{Yb02} \mathrm{O} 14$ & $142.2(10)$ & $\mathrm{O} 2$ & Yb08 C61 & 71.8( \\
\hline O18 & Yb02 O9 & $72.4(9)$ & $\mathrm{O} 2$ & Yb08 O3 & $73.3(11)$ \\
\hline $\mathrm{O} 18$ & Yb02 C33 & $17.2(10)$ & $\mathrm{O} 12 \mathrm{~S}$ & Yb08 Yb05 & $142 .($ \\
\hline 18 & Yb02 C17 & $68.0(12)$ & $\mathrm{O} 12 \mathrm{~S}$ & Yb08 Yb06 & 146.0 \\
\hline $\mathrm{O} 36$ & Yb02 Yb01 & $66.8(6)$ & $\mathrm{O} 12$ & Yb08 Yb07 & $147.8(9)$ \\
\hline 36 & Yb02 Yb03 & $116.6(6)$ & $\mathrm{O} 12 \mathrm{~S}$ & Yb08 C61 & 91(2) \\
\hline $\mathrm{O} 36$ & Yb02 O7 & $78.8(8)$ & $\mathrm{O} 33$ & Yb08 Yb05 & $70.2(9)$ \\
\hline $\mathrm{O} 36$ & Yb02 O6 & $80.6(8)$ & $\mathrm{O} 33$ & Yb08 Yb06 & 120. \\
\hline $\mathrm{O} 36$ & Yb02 O5 & $147.3(8)$ & $\mathrm{O} 33$ & Yb08 Yb07 & $117.7(9)$ \\
\hline $\mathrm{O} 36$ & Yb02 O18 & 97.3(9) & $\mathrm{O} 33$ & Yb08 O1 & $74.4(12)$ \\
\hline $\mathrm{O} 36$ & Yb02 O10 & $77.6(10)$ & $\mathrm{O} 33$ & Yb08 O2 & $80.1(12)$ \\
\hline $\mathrm{O} 36$ & Yb02 O14 & $99.7(10)$ & $\mathrm{O} 33$ & Yb08 O12S & $71.9(14)$ \\
\hline $\mathrm{O} 36$ & Yb02 O9 & $134.4(10)$ & $\mathrm{O} 33$ & Yb08 O15 & $119(2)$ \\
\hline $\mathrm{O} 36$ & Yb02 C33 & $106.6(10)$ & $\mathrm{O} 33$ & Yb08 C61 & $116(3)$ \\
\hline $\mathrm{O} 36$ & Yb02 C17 & $108.6(13)$ & $\mathrm{O} 33$ & Yb08 O3 & $148.2(12)$ \\
\hline $\mathrm{O} 10$ & Yb02 Yb01 & $144.4(7)$ & O15 & Yb08 Yb05 & $118.0(11)$ \\
\hline $\mathrm{O} 10$ & Yb02 Yb03 & $143.9(7)$ & O15 & Yb08 Yb06 & $67.9(15)$ \\
\hline $\mathrm{O} 10$ & Yb02 O9 & $56.8(10)$ & O15 & Yb08 Yb07 & $115.7(19)$ \\
\hline
\end{tabular}




\begin{tabular}{|c|c|c|c|c|c|c|}
\hline $\mathrm{O} 10$ & Yb02 C33 & $90.2(11)$ & O15 & Yb08 & O1 & $83.5(13)$ \\
\hline $\mathrm{O} 10$ & Yb02 C17 & $31.1(12)$ & O15 & Yb08 & $\mathrm{O} 2$ & $145.7(18)$ \\
\hline 14 & Yb02 Yb01 & $106.7(7)$ & O15 & Yb08 & O12S & $78.5(19)$ \\
\hline 14 & $\mathrm{Yb} 02 \mathrm{Yb} 03$ & $127.6(7)$ & O15 & Yb08 & C61 & $116(2)$ \\
\hline 14 & Yb02 O7 & $140.7(8)$ & O15 & Yb08 & $\mathrm{O} 3$ & $78(2)$ \\
\hline 14 & Yb02 O6 & $71.5(9)$ & C61 & Yb08 & Yb05 & $108.8(17)$ \\
\hline 014 & Yb02 O5 & $92.3(9)$ & C61 & Yb08 & Yb06 & $108(3)$ \\
\hline 14 & Yb02 O10 & $77.2(10)$ & C61 & Yb08 & Yb07 & $57(2)$ \\
\hline 14 & Yb02 O9 & $71.5(11)$ & $\mathrm{O} 30$ & Yb08 & Yb05 & $123.3(15)$ \\
\hline 14 & Yb02 C33 & $147.6(11)$ & $\mathrm{O} 30$ & Yb08 & Yb06 & $129(2)$ \\
\hline 14 & Yb02 C17 & $74.7(12)$ & $\mathrm{O} 30$ & Yb08 & Yb07 & $79(2)$ \\
\hline O9 & Yb02 Yb01 & $158.7(7)$ & $\mathrm{O} 30$ & Yb08 & $\mathrm{O} 1$ & $160.1(17)$ \\
\hline j9 & $\mathrm{Yb} 02 \mathrm{Yb} 03$ & $102.3(8)$ & $\mathrm{O} 30$ & Yb08 & $\mathrm{O} 2$ & $85.7(18)$ \\
\hline O9 & Yb02 C33 & $76.5(10)$ & $\mathrm{O} 30$ & Yb08 & $\mathrm{O} 12 \mathrm{~S}$ & $69(2)$ \\
\hline O9 & Yb02 C17 & $25.9(11)$ & $\mathrm{O} 30$ & Yb08 & $\mathrm{O} 33$ & $103(2)$ \\
\hline C33 & Yb02 Yb01 & $100.9(8)$ & $\mathrm{O} 30$ & Yb08 & O15 & $113.9(19)$ \\
\hline 33 & Yb02 Yb03 & $54.5(8)$ & $\mathrm{O} 30$ & Yb08 & C61 & $22(3)$ \\
\hline $\mathrm{C} 17$ & Yb02 Yb01 & $175.4(11)$ & $\mathrm{O} 30$ & Yb08 & $\mathrm{O} 3$ & $92(2)$ \\
\hline 17 & $\mathrm{Yb} 02 \mathrm{Yb} 03$ & $121.7(11)$ & $\mathrm{O} 3$ & Yb08 & Yb05 & 78.1(7) \\
\hline $\mathrm{C} 17$ & Yb02 C33 & $79.2(13)$ & $\mathrm{O} 3$ & Yb08 & Yb06 & $37.3(5)$ \\
\hline $\mathrm{rb} 02$ & Yb03 Yb01 & $59.28(4)$ & $\mathrm{O} 3$ & Yb08 & Yb07 & $37.9(7)$ \\
\hline Yb04 & Yb03 Yb01 & $60.51(5)$ & $\mathrm{O} 3$ & Yb08 & $\mathrm{O} 1$ & $81.9(9)$ \\
\hline Yb04 & Yb03 Yb02 & $60.23(5)$ & $\mathrm{O} 3$ & Yb08 & O12S & $139.9(12)$ \\
\hline O7 & Yb03 Yb01 & $34.8(4)$ & $\mathrm{O} 3$ & Yb08 & C61 & $72(3)$ \\
\hline $\mathrm{O} 7$ & Yb03 Yb02 & $37.8(4)$ & Yb01 & O7 & Yb02 & $106.2(8)$ \\
\hline $\mathrm{O} 7$ & Yb03 Yb04 & $77.3(5)$ & Yb01 & O7 & Yb03 & $109.3(7)$ \\
\hline O7 & Yb03 O8 & $68.7(6)$ & Yb03 & O7 & Yb02 & $104.5(7)$ \\
\hline $\mathrm{O} 7$ & Yb03 O5 & $72.0(7)$ & Yb01 & O8 & Yb03 & $106.4(7)$ \\
\hline $\mathrm{O} 7$ & Yb03 O6S & $135.2(9)$ & Yb04 & $\mathrm{O} 8$ & Yb01 & $108.2(7)$ \\
\hline $\mathrm{O} 7$ & Yb03 C33 & $64.3(9)$ & Yb04 & $\mathrm{O} 8$ & Yb03 & $104.6(7)$ \\
\hline $\mathrm{O} 7$ & Yb03 O7S & $130.9(13)$ & Yb01 & O6 & Yb02 & $105.6(8)$ \\
\hline O8 & Yb03 Yb01 & $36.5(4)$ & Yb01 & O6 & Yb04 & $107.8(8)$ \\
\hline O8 & Yb03 Yb02 & $76.4(4)$ & Yb02 & O6 & Yb04 & $102.1(7)$ \\
\hline O8 & Yb03 Yb04 & $37.2(5)$ & Yb05 & $\mathrm{O} 4$ & Yb06 & $110.1(11)$ \\
\hline O8 & Yb03 O6S & $137.8(9)$ & Yb05 & $\mathrm{O} 4$ & Yb07 & $113.5(12)$ \\
\hline O8 & Yb03 C33 & $128.7(10)$ & Yb07 & $\mathrm{O} 4$ & Yb06 & $96.7(9)$ \\
\hline O8 & Yb03 O7S & $127.9(15)$ & Yb05 & O1 & Yb06 & $92.1(9)$ \\
\hline $\mathrm{O} 5$ & Yb03 Yb01 & $78.5(5)$ & Yb08 & $\mathrm{O} 1$ & Yb05 & 107.1(11) \\
\hline
\end{tabular}




\begin{tabular}{|c|c|c|c|c|c|c|}
\hline O5 & Yb03 Yb02 & $36.9(5)$ & Yb08 & $\mathrm{O} 1$ & Yb06 & $96.1(9)$ \\
\hline O5 & Yb03 Yb04 & $38.7(5)$ & Yb02 & O5 & Yb03 & $105.3(7)$ \\
\hline 05 & Yb03 O8 & $72.5(7)$ & Yb02 & O5 & $\mathrm{Yb} 04$ & $105.2(8)$ \\
\hline 55 & $\mathrm{Yb} 03 \mathrm{O}$ - & $140.2(9)$ & Yb03 & O5 & Yb04 & $103.1(8)$ \\
\hline$)^{2}$ & Yb03 C33 & $74.6(10)$ & C53 & $\mathrm{O} 35$ & Yb01 & $122(2)$ \\
\hline 05 & Yb03 O7S & $72.0(14)$ & C 33 & $\mathrm{O} 18$ & Yb02 & $129(3)$ \\
\hline $\mathrm{O} 22$ & Yb03 Yb01 & $69.8(5)$ & C49 & $\mathrm{O} 26$ & Yb01 & $93(2)$ \\
\hline 22 & Yb03 Yb02 & $120.0(5)$ & C53 & O36 & Yb02 & $138(2)$ \\
\hline 022 & Yb03 Yb04 & $118.4(6)$ & $\mathrm{C} 25$ & $\mathrm{O} 21$ & Yb01 & $139(2)$ \\
\hline $\mathrm{j} 22$ & Yb03 O7 & $82.2(6)$ & C49 & $\mathrm{O} 25$ & Yb01 & $92(2)$ \\
\hline $\mathrm{O} 22$ & Yb03 O8 & $81.3(7)$ & $\mathrm{C} 40$ & $\mathrm{O} 20$ & Yb01 & $136(3)$ \\
\hline $\mathrm{O} 22$ & Yb03 O5 & $148.3(7)$ & $\mathrm{C} 25$ & $\mathrm{O} 22$ & Yb03 & $137(2)$ \\
\hline $\mathrm{O} 22$ & $\mathrm{Yb} 03 \mathrm{O} 6 \mathrm{~S}$ & $71.6(9)$ & $\mathrm{C} 24$ & O11 & $\mathrm{Yb}^{2} 5^{1}$ & $86(3)$ \\
\hline $\mathrm{O} 22$ & Yb03 C33 & $110.7(9)$ & $\mathrm{C} 16$ & $\mathrm{O} 34$ & Yb05 & $147(4)$ \\
\hline $\mathrm{O} 22$ & Yb03 O7S & $139.7(14)$ & $\mathrm{C} 51$ & C50 & C49 & $120(3)$ \\
\hline $66 \mathrm{~S}$ & Yb03 Yb01 & $141.2(7)$ & $\mathrm{C} 52^{2}$ & $\mathrm{C} 50$ & C51 & $122(3)$ \\
\hline O6S & Yb03 Yb02 & $145.3(7)$ & $\mathrm{C} 52^{2}$ & $\mathrm{C} 50$ & $\mathrm{C} 49$ & $117(4)$ \\
\hline O6S & Yb03 Yb04 & $147.3(7)$ & $\mathrm{C} 17$ & O10 & $\mathrm{Yb} 02$ & $88(2)$ \\
\hline O6S & Yb03 C33 & $91.5(12)$ & Yb07 & $\mathrm{O} 2$ & Yb05 & $101.6(12)$ \\
\hline $\mathrm{O} 6 \mathrm{~S}$ & $\mathrm{Yb} 03 \mathrm{O}$ O7S & $68.3(15)$ & Yb08 & $\mathrm{O} 2$ & Yb05 & $106.7(13)$ \\
\hline $\mathrm{O} 17$ & Yb03 Yb01 & $114.8(6)$ & Yb08 & $\mathrm{O} 2$ & Yb07 & $106.6(13)$ \\
\hline O17 & Yb03 Yb02 & $72.5(6)$ & C8 & O14 & Yb02 & $137(3)$ \\
\hline $\mathrm{O} 17$ & Yb03 Yb04 & $126.6(7)$ & $\mathrm{C} 17$ & O9 & Yb02 & $88(3)$ \\
\hline $\mathrm{O} 17$ & Yb03 O7 & $80.3(8)$ & $\mathrm{C} 24$ & $\mathrm{O} 12$ & $\mathrm{Yb}^{2} 5^{1}$ & $92(4)$ \\
\hline O17 & Yb03 O8 & $147.2(7)$ & $\mathrm{C} 41$ & O39 & Yb06 & $90(3)$ \\
\hline $\mathrm{O} 17$ & Yb03 O5 & $88.4(9)$ & $\mathrm{C} 31$ & $\mathrm{C} 26$ & $\mathrm{C} 25$ & $119(3)$ \\
\hline O17 & $\mathrm{Yb} 03 \mathrm{O} 22$ & $105.5(9)$ & C31 & $\mathrm{C} 26$ & $\mathrm{C} 27$ & $123(4)$ \\
\hline O17 & Yb03 O6S & $72.9(10)$ & $\mathrm{C} 27$ & $\mathrm{C} 26$ & $\mathrm{C} 25$ & $117(3)$ \\
\hline $\mathrm{O} 17$ & Yb03 C33 & $18.7(11)$ & $\mathrm{C} 41$ & $\mathrm{O} 40$ & Yb06 & $87(3)$ \\
\hline O17 & $\mathrm{Yb} 03 \mathrm{O} 7 \mathrm{~S}$ & $66.4(15)$ & C 34 & C35 & C36 & $120(4)$ \\
\hline C33 & Yb03 Yb01 & $99.0(8)$ & C54 & C55 & $\mathrm{C} 55^{3}$ & $123(2)$ \\
\hline $\mathrm{C} 33$ & Yb03 Yb02 & $54.0(9)$ & C55 & C54 & C53 & $128(4)$ \\
\hline $\mathrm{C} 33$ & Yb03 Yb04 & $110.9(9)$ & $\mathrm{C} 55$ & C54 & $\mathrm{C} 56$ & $120(3)$ \\
\hline $\mathrm{O} 28$ & Yb03 Yb01 & $115.3(9)$ & C56 & C54 & C53 & $112(3)$ \\
\hline $\mathrm{O} 28$ & Yb03 Yb02 & $127.8(12)$ & $\mathrm{O} 21$ & $\mathrm{C} 25$ & $\mathrm{O} 22$ & $120(3)$ \\
\hline $\mathrm{O} 28$ & Yb03 Yb04 & $72.6(9)$ & $\mathrm{O} 21$ & $\mathrm{C} 25$ & $\mathrm{C} 26$ & $121(3)$ \\
\hline $\mathrm{O} 28$ & Yb03 O7 & 147.1(10) & $\mathrm{O} 22$ & $\mathrm{C} 25$ & $\mathrm{C} 26$ & $119(3)$ \\
\hline $\mathrm{O} 28$ & Yb03 O8 & $79.3(10)$ & $\mathrm{O} 35$ & $\mathrm{C} 53$ & Yb01 & $37.1(15)$ \\
\hline
\end{tabular}




\begin{tabular}{|c|c|c|c|c|c|c|}
\hline 88 & $03 \mathrm{O}$ & & 35 & C53 & C54 & \\
\hline & $\mathrm{Yb} 03 \mathrm{O} 22$ & (15) & $\mathrm{O} 36$ & C53 & Yb01 & $91(2)$ \\
\hline & Yb03 O6S & 74.9 & 36 & C53 & $\mathrm{O} 35$ & $27(3)$ \\
\hline & Yb03 017 & 128. & 036 & C53 & C54 & $1(4)$ \\
\hline & Yb03 C33 & 139 & C54 & C53 & Yb01 & $8(3)$ \\
\hline & b03 O7S & 6 & $\mathrm{C} 26$ & $\mathrm{C} 31$ & $\mathrm{C} 30$ & $20(4)$ \\
\hline & b03 Yb01 & & C35 & C34 & C33 & 1(4) \\
\hline & Yb03 Yb02 & & C39 & C34 & C35 & $116(3)$ \\
\hline & Yb03 Yb04 & & C39 & $\mathrm{C} 34$ & C33 & $23(4)$ \\
\hline & Yb03 C33 & 74. & $\mathrm{C} 43$ & C42 & $\mathrm{C} 41$ & $7(4)$ \\
\hline & Yb04 Y & 59.2 & $\mathrm{C} 43$ & $\mathrm{C} 42$ & $\mathrm{C} 44$ & $124(5)$ \\
\hline & $004 \mathrm{Y}$ & & $\mathrm{C} 44$ & $\mathrm{C} 42$ & $\mathrm{C} 41$ & $16(5)$ \\
\hline & Yb04 & & C33 & O17 & Yb03 & $127(3)$ \\
\hline & b04 Y & & $\mathrm{Yb} 02$ & C33 & Yb03 & $71.5(8$ \\
\hline & b04 & & O18 & $\mathrm{C} 33$ & $\mathrm{Yb} 02$ & \\
\hline & b04 & & $\mathrm{O} 18$ & $\mathrm{C} 33$ & Yb03 & $105(3)$ \\
\hline & Yb04 & & O18 & C33 & C34 & 4(4) \\
\hline & Yb04 & 73.1 & O18 & C33 & O17 & $139(4)$ \\
\hline & Yb04 O8S & 136 & C34 & C33 & Yb02 & $8(3)$ \\
\hline & Yb04 C & 136 & C34 & C33 & $\mathrm{Yb} 03$ & 139(3) \\
\hline & Yb04 Y & 34.8 & $\mathrm{O} 17$ & $\mathrm{C} 33$ & Yb02 & $105(2)$ \\
\hline & Yb04 Y & & O17 & C33 & $\mathrm{Yb} 03$ & $33.9(1$ \\
\hline & $\mathrm{Yb} 04 \mathrm{Y}$ & 78. & O17 & $\mathrm{C} 33$ & $\mathrm{C} 34$ & $107(4)$ \\
\hline & Yb04 C & 136 & C52 & $\mathrm{C} 51$ & $\mathrm{C} 50$ & $116(4)$ \\
\hline & Yb04 C & 120 & $\mathrm{O} 26$ & $\mathrm{C} 49$ & Yb01 & $60.7(17$ \\
\hline & Yb04 & & $\mathrm{O} 26$ & C49 & $\mathrm{O} 25$ & 121 \\
\hline & Yb04 Y & & $\mathrm{O} 26$ & C49 & C50 & $119(3)$ \\
\hline & Yb04 Yb03 & 38.3 & $\mathrm{O} 25$ & C49 & Yb01 & $61.0(1$ \\
\hline & Yb04 O6 & 73.0 & $\mathrm{O} 25$ & $\mathrm{C} 49$ & $\mathrm{C} 50$ & $118(3)$ \\
\hline 5 & Yb04 O8S & 139.0( & $\mathrm{C} 50$ & $\mathrm{C} 49$ & Yb01 & $176(3)$ \\
\hline & Yb04 O9S & 69.9 & $\mathrm{C} 46$ & $\mathrm{C} 47$ & $\mathrm{C} 48$ & $121(5)$ \\
\hline & Yb04 Y & $142.6(8)$ & C26 & $\mathrm{C} 27$ & $\mathrm{C} 28$ & $113(4)$ \\
\hline 885 & Yb04 Yb02 & $146.6(9)$ & N5 & $\mathrm{C} 27$ & $\mathrm{C} 26$ & $133(5)$ \\
\hline & Yb04 Y & 145.1 & N5 & $\mathrm{C} 27$ & $\mathrm{C} 28$ & $115(4)$ \\
\hline & Yb04 O9S & $69.9(16)$ & $\mathrm{C} 50^{2}$ & C52 & C51 & $121(4)$ \\
\hline & Yb04 Yb01 & $70.9(8)$ & C37 & C38 & C39 & $118(4)$ \\
\hline & Yb04 Y & & $\mathrm{C} 16$ & $\mathrm{O} 33$ & Yb08 & $143(4)$ \\
\hline & Yb04 & $117.0(8)$ & $\mathrm{C} 40$ & O19 & Yb04 & $133(2)$ \\
\hline
\end{tabular}




\begin{tabular}{|c|c|c|c|c|c|c|}
\hline O19 & Yb04 O8 & $79.0(9)$ & C57 & O38 & Yb05 & $133(4)$ \\
\hline O19 & Yb04 O6 & $84.3(8)$ & $\mathrm{C} 1$ & O16 & Yb06 & $136(4)$ \\
\hline O19 & Yb04 O5 & $148.8(9)$ & $\mathrm{C} 32$ & $\mathrm{O} 23$ & Yb07 & $140(3)$ \\
\hline 19 & Yb04 O8S & $71.9(12)$ & C57 & $\mathrm{O} 37$ & Yb06 & $139(4)$ \\
\hline O19 & Yb04 O27 & $103.4(12)$ & $\mathrm{C} 45$ & $\mathrm{O} 27$ & Yb04 & $141(2)$ \\
\hline O19 & Yb04 O9S & $141.2(16)$ & O11 & $\mathrm{C} 24$ & ${\mathrm{Yb} 05^{1}}^{1}$ & $65(3)$ \\
\hline $\mathrm{O} 27$ & Yb04 Yb01 & $118.3(10)$ & O11 & $\mathrm{C} 24$ & $\mathrm{C} 21$ & $122(5)$ \\
\hline $\mathrm{O} 27$ & Yb04 Yb02 & $122.9(7)$ & $\mathrm{O} 12$ & $\mathrm{C} 24$ & $\mathrm{Yb} 05^{1}$ & $61(3)$ \\
\hline 27 & Yb04 Yb03 & $70.8(7)$ & O12 & $\mathrm{C} 24$ & O11 & $127(5)$ \\
\hline 27 & Yb04 O8 & $82.2(11)$ & $\mathrm{O} 12$ & $\mathrm{C} 24$ & $\mathrm{C} 21$ & $112(6)$ \\
\hline $\mathrm{O} 27$ & Yb04 O6 & $148.2(9)$ & $\mathrm{C} 21$ & $\mathrm{C} 24$ & $\mathrm{Yb} 05^{1}$ & $172(5)$ \\
\hline $\mathrm{O} 27$ & Yb04 O5 & $86.5(9)$ & $\mathrm{C} 8$ & O13 & Yb04 & $143(3)$ \\
\hline $\mathrm{O} 27$ & Yb04 O8S & $74.3(10)$ & O38 & C57 & C58 & $111(6)$ \\
\hline 27 & Yb04 O9S & $73(3)$ & O37 & C57 & O38 & $125(5)$ \\
\hline 13 & Yb04 Yb01 & $108.4(8)$ & O37 & C57 & C58 & $123(5)$ \\
\hline 13 & Yb04 Yb02 & $69.9(9)$ & $\mathrm{C} 1$ & O15 & Yb08 & $137(4)$ \\
\hline 13 & Yb04 Yb03 & $126.5(10)$ & C59 & C58 & $\mathrm{C} 57$ & $124(4)$ \\
\hline 12 & Yb04 O8 & $142.2(9)$ & C59 & C58 & C60 & $123(3)$ \\
\hline 13 & Yb04 O6 & $73.9(9)$ & C60 & C58 & C57 & $112(4)$ \\
\hline $\mathrm{O} 13$ & Yb04 O5 & $89.6(12)$ & $\mathrm{C} 45$ & $\mathrm{O} 28$ & Yb03 & $140(4)$ \\
\hline $\mathrm{O} 13$ & Yb04 O8S & $77.5(12)$ & C6 & $\mathrm{C} 5$ & $\mathrm{C} 4$ & $115(5)$ \\
\hline $\mathrm{O} 13$ & Yb04 O19 & $104.6(13)$ & $\mathrm{C} 6$ & $\mathrm{C} 5$ & $\mathrm{C} 8$ & $125(6)$ \\
\hline $\mathrm{O} 13$ & Yb04 O27 & $131.2(14)$ & $\mathrm{C} 8$ & C5 & $\mathrm{C} 4$ & $120(5)$ \\
\hline $\mathrm{O} 13$ & Yb04 O9S & $60(2)$ & C58 & C59 & $\mathrm{C} 60^{1}$ & $121(4)$ \\
\hline O9S & Yb04 Yb01 & $145.8(15)$ & $\mathrm{C} 32^{4}$ & $\mathrm{C} 29$ & $\mathrm{C} 30$ & $118(4)$ \\
\hline O9S & Yb04 Yb02 & $87.2(16)$ & $\mathrm{C} 28$ & $\mathrm{C} 29$ & $\mathrm{C} 30$ & $117(4)$ \\
\hline O9S & Yb04 Yb03 & $98.6(17)$ & $\mathrm{C} 28$ & $\mathrm{C} 29$ & $\mathrm{C} 32^{4}$ & $125(4)$ \\
\hline Yb06 & Yb05 Yb07 & $60.02(8)$ & O39 & C41 & Yb06 & $63(3)$ \\
\hline Yb06 & Yb05 Yb08 & $60.86(9)$ & O39 & $\mathrm{C} 41$ & O40 & $126(5)$ \\
\hline Yb07 & Yb05 Yb08 & $60.01(11)$ & O39 & C41 & $\mathrm{C} 42$ & $117(5)$ \\
\hline $\mathrm{O} 4$ & Yb05 Yb06 & $39.6(8)$ & $\mathrm{O} 40$ & $\mathrm{C} 41$ & Yb06 & $65(3)$ \\
\hline $\mathrm{O} 4$ & Yb05 Yb07 & $37.2(8)$ & $\mathrm{O} 40$ & $\mathrm{C} 41$ & $\mathrm{C} 42$ & $114(5)$ \\
\hline $\mathrm{O} 4$ & Yb05 Yb08 & $80.4(8)$ & $\mathrm{C} 42$ & C41 & Yb06 & $175(4)$ \\
\hline $\mathrm{O} 4$ & Yb05 O1 & $84.9(10)$ & C31 & C30 & C29 & $119(4)$ \\
\hline $\mathrm{O} 4$ & Yb05 O11 ${ }^{1}$ & $77.8(12)$ & C34 & C39 & C38 & $124(3)$ \\
\hline $\mathrm{O} 4$ & Yb05 O34 & $150.0(12)$ & O10 & $\mathrm{C} 17$ & $\mathrm{Yb02}$ & $60.9(18)$ \\
\hline $\mathrm{O} 4$ & Yb05 O2 & $74.0(11)$ & $\mathrm{O} 10$ & $\mathrm{C} 17$ & $\mathrm{C} 18$ & $114(4)$ \\
\hline $\mathrm{O} 4$ & Yb05 O12 & $132.8(13)$ & O9 & $\mathrm{C} 17$ & $\mathrm{Yb} 02$ & $66(2)$ \\
\hline
\end{tabular}




\begin{tabular}{|c|c|c|c|c|c|c|}
\hline 4 & 05038 & & D9 & C17 & $\mathrm{O} 10$ & \\
\hline 4 & $\mathrm{~b} 05 \mathrm{C} 24^{1}$ & $106.4(17)$ & O9 & $\mathrm{C} 17$ & $\mathrm{C} 18$ & 19(4) \\
\hline & $005 \mathrm{O} 24$ & 90.8 & $\mathrm{C} 18$ & $\mathrm{C} 17$ & Yb02 & $173(4)$ \\
\hline & $005 \mathrm{Yb}$ & 48.1 & $\mathrm{C} 3$ & $\mathrm{C} 4$ & $\mathrm{C} 5$ & (5) \\
\hline & b05 Yl & is & C6 & $\mathrm{C} 7$ & $\mathrm{C} 2$ & (5) \\
\hline & $005 \mathrm{YbC}$ & 36.0 & $\mathrm{O} 20$ & C40 & O19 & $25(3)$ \\
\hline & $\mathrm{Yb} 05 \mathrm{O}^{1} 1^{1}$ & $137.3(10)$ & $\mathrm{O} 20$ & $\mathrm{C} 40$ & $\mathrm{C} 37^{5}$ & 123(4) \\
\hline & rb05 O2 & $71.2(10)$ & O19 & $\mathrm{C} 40$ & $\mathrm{C} 37^{5}$ & $112(4)$ \\
\hline 1 & $\mathrm{~b} 05 \mathrm{O} 12^{1}$ & 131.8 & $\mathrm{O} 27$ & $\mathrm{C} 45$ & $\mathrm{O} 28$ & $115(4)$ \\
\hline 1 & b05 O38 & 71.1 & $\mathrm{O} 27$ & $\mathrm{C} 45$ & $\mathrm{C} 46$ & $118(4)$ \\
\hline & $\mathrm{Yb} 05 \mathrm{C} 24^{1}$ & $142.0(12)$ & $\mathrm{O} 28$ & $\mathrm{C} 45$ & $\mathrm{C} 46$ & $126(5)$ \\
\hline $11^{1}$ & Yb05 Yb06 & $99.4(9)$ & C54 & C56 & $\mathrm{C} 56^{3}$ & $115(3)$ \\
\hline & b05 & 102.8 & $\mathrm{C} 5$ & C6 & C7 & $129(6)$ \\
\hline $1^{1}$ & b05 Yb08 & 158.0 & C58 & C60 & $\mathrm{C} 59^{1}$ & $113(4)$ \\
\hline $1^{1}$ & $005 \mathrm{C} 24^{1}$ & 28.7 & $\mathrm{C} 42$ & $\mathrm{C} 43$ & $\mathrm{C} 43^{3}$ & 117(4) \\
\hline 34 & b05 & 119.2 & $\mathrm{C} 42$ & $\mathrm{C} 44$ & $\mathrm{C} 44^{3}$ & $116(4)$ \\
\hline & b05 Yb07 & 120 & N1 & C44 & $\mathrm{C} 42$ & $127(7)$ \\
\hline 34 & Yb05 Y & $69.6(9)$ & N1 & $\mathrm{C} 44$ & $\mathrm{C} 44^{3}$ & $116(4)$ \\
\hline 34 & Yb05 O1 & $71.2(1$ & $\mathrm{O} 23$ & $\mathrm{C} 32$ & $\mathrm{C} 29^{6}$ & $119(4)$ \\
\hline 34 & Yb05 O11 ${ }^{1}$ & $132.1(13)$ & $\mathrm{O} 24$ & $\mathrm{C} 32$ & $\mathrm{O} 23$ & $120(4)$ \\
\hline 34 & $\mathrm{Yb} 05 \mathrm{O} 2$ & $81.2(11)$ & $\mathrm{O} 24$ & $\mathrm{C} 32$ & $\mathrm{C} 29^{6}$ & $120(4)$ \\
\hline 34 & $\mathrm{Yb} 05 \mathrm{O} 12^{1}$ & $77.2(13)$ & C47 & C46 & $\mathrm{C} 45$ & $122(5)$ \\
\hline 34 & Yb05 O38 & $93.8(15)$ & C47 & C46 & $\mathrm{C} 48^{7}$ & $132(6)$ \\
\hline 34 & $\mathrm{Yb} 05 \mathrm{C} 24^{1}$ & 103.6( & $\mathrm{C} 45$ & $\mathrm{C} 46$ & $\mathrm{C} 48^{7}$ & $107(5)$ \\
\hline 34 & Yb05 O24 & 98.4( & $\mathrm{C} 2$ & $\mathrm{C} 3$ & $\mathrm{C} 4$ & $132(6)$ \\
\hline & Yb05 Y & 78.4 & $\mathrm{C} 2$ & $\mathrm{C} 3$ & $\mathrm{~N}$ & $127(7)$ \\
\hline & Yb05 Yb07 & 38 & N3 & $\mathrm{C} 3$ & $\mathrm{C} 4$ & $101(6)$ \\
\hline 2 & Yb05 & $35.5(8)$ & $\mathrm{C} 27$ & $\mathrm{C} 28$ & N6 & $111(5)$ \\
\hline 2 & Yb05 O11 ${ }^{1}$ & 137.1(11) & $\mathrm{C} 29$ & $\mathrm{C} 28$ & $\mathrm{C} 27$ & $128(4)$ \\
\hline 2 & $\mathrm{Yb} 05 \mathrm{O} 12^{1}$ & $138.0(11)$ & $\mathrm{C} 29$ & $\mathrm{C} 28$ & N6 & $121(5)$ \\
\hline & $\mathrm{Yb} 05 \mathrm{C} 24^{1}$ & 146.5( & O14 & $\mathrm{C} 8$ & $\mathrm{C} 5$ & $120(4)$ \\
\hline 12 & b05 Yb06 & 143.5 & $\mathrm{O} 13$ & $\mathrm{C} 8$ & $\mathrm{O} 14$ & $120(4)$ \\
\hline $12^{1}$ & Yb05 Yb07 & $143.3(7)$ & O13 & $\mathrm{C} 8$ & $\mathrm{C} 5$ & $120(4)$ \\
\hline $12^{1}$ & b05 Yb08 & $146.8(10)$ & $\mathrm{C} 32$ & $\mathrm{O} 24$ & Yb05 & $137(3)$ \\
\hline Ol & Yb05 $011^{1}$ & $55.2(13)$ & C38 & C37 & $\mathrm{C} 40^{8}$ & $124(4)$ \\
\hline $\mathrm{O} 12^{1}$ & $\mathrm{Yb} 05 \mathrm{C} 24^{1}$ & $26.5(15)$ & C38 & C37 & C36 & $121(4)$ \\
\hline 28 & Yb05 Yb06 & $71.0(11)$ & $\mathrm{C} 36$ & $\mathrm{C} 37$ & $\mathrm{C} 40^{8}$ & $115(4)$ \\
\hline 38 & Yb05 Yb07 & $129.3(13)$ & $\mathrm{C} 7$ & $\mathrm{C} 2$ & $\mathrm{C} 1$ & $125(5)$ \\
\hline
\end{tabular}




\begin{tabular}{|c|c|c|c|c|c|c|}
\hline O38 & Yb05 Yb08 & $107.1(7)$ & C3 & $\mathrm{C} 2$ & $\mathrm{C} 7$ & $114(5)$ \\
\hline O38 & Yb05 O11 ${ }^{1}$ & $72.0(10)$ & C3 & $\mathrm{C} 2$ & $\mathrm{C} 1$ & $121(6)$ \\
\hline $\mathrm{O} 38$ & Yb05 O2 & $141.6(11)$ & $\mathrm{C} 37$ & $\mathrm{C} 36$ & $\mathrm{C} 35$ & $120(4)$ \\
\hline O38 & Yb05 O12 & $75.8(12)$ & O16 & $\mathrm{C} 1$ & Yb06 & $29(3)$ \\
\hline $\mathrm{O} 38$ & Yb05 C24 ${ }^{1}$ & $71.8(12)$ & O16 & $\mathrm{C} 1$ & $\mathrm{O} 15$ & $125(5)$ \\
\hline $\mathrm{C} 24^{1}$ & Yb05 Yb06 & $123.9(13)$ & $\mathrm{O} 16$ & $\mathrm{C} 1$ & $\mathrm{C} 2$ & $122(6)$ \\
\hline $\mathrm{C} 24^{1}$ & Yb05 Yb07 & $126.1(14)$ & $\mathrm{O} 15$ & $\mathrm{C} 1$ & Yb06 & $95(3)$ \\
\hline $\mathrm{C} 24^{1}$ & Yb05 Yb08 & $173.1(15)$ & O15 & $\mathrm{C} 1$ & $\mathrm{C} 2$ & $111(6)$ \\
\hline 24 & Yb05 Yb06 & $128.6(10)$ & $\mathrm{C} 2$ & $\mathrm{C} 1$ & Yb06 & $149(4)$ \\
\hline $\mathrm{O} 24$ & Yb05 Yb07 & $71.4(8)$ & O34 & $\mathrm{C} 16$ & $\mathrm{C} 13^{9}$ & $129(10)$ \\
\hline 24 & Yb05 Yb08 & $108.7(7)$ & O33 & $\mathrm{C} 16$ & $\mathrm{O} 34$ & $109(5)$ \\
\hline $\mathrm{O} 24$ & Yb05 O1 & $144.7(10)$ & $\mathrm{O} 33$ & $\mathrm{C} 16$ & $\mathrm{C} 13^{9}$ & $121(10)$ \\
\hline $\mathrm{O} 24$ & Yb05 O11 ${ }^{1}$ & $74.9(11)$ & $\mathrm{C} 15$ & $\mathrm{C} 14$ & $\mathrm{C} 13$ & 120.0 \\
\hline 24 & Yb05 O2 & $73.9(10)$ & $\mathrm{C} 15$ & $\mathrm{C} 14$ & $\mathrm{~N} 2$ & $114(5)$ \\
\hline $\mathrm{O} 24$ & Yb05 O12 1 & $74.2(10)$ & $\mathrm{C} 13$ & $\mathrm{C} 14$ & N2 & $125(5)$ \\
\hline 24 & Yb05 O38 & $144.1(9)$ & $\mathrm{C} 14$ & $\mathrm{C} 15$ & $\mathrm{C} 10$ & 120.0 \\
\hline $\mathrm{O} 24$ & $\mathrm{Yb} 05 \mathrm{C} 24^{1}$ & $72.6(12)$ & $\mathrm{C} 11$ & $\mathrm{C} 10$ & $\mathrm{C} 15$ & 120.0 \\
\hline Yb05 & Yb06 Yb07 & $60.27(8)$ & C9 & $\mathrm{C} 10$ & $\mathrm{C} 15$ & $120(4)$ \\
\hline $\mathrm{O} 4$ & Yb06 Yb05 & $30.3(6)$ & C9 & $\mathrm{C} 10$ & & $120(4)$ \\
\hline $\mathrm{O} 4$ & Yb06 Yb07 & $41.0(6)$ & $\mathrm{C} 10$ & $\mathrm{C} 11$ & $\mathrm{C} 12$ & 120.0 \\
\hline $\mathrm{O} 4$ & Yb06 O1 & $68.0(8)$ & $\mathrm{C} 13$ & $\mathrm{C} 12$ & $\mathrm{C} 11$ & 120.0 \\
\hline $\mathrm{O} 4$ & Yb06 C41 & $109.9(12)$ & $\mathrm{C} 14$ & $\mathrm{C} 13$ & $\mathrm{C} 16^{10}$ & $120(4)$ \\
\hline $\mathrm{O} 4$ & Yb06 C1 & $133.8(13)$ & $\mathrm{C} 12$ & $\mathrm{C} 13$ & $\mathrm{C} 16^{10}$ & $120(4)$ \\
\hline $\mathrm{O} 1$ & Yb06 Yb05 & $39.8(6)$ & $\mathrm{C} 12$ & $\mathrm{C} 13$ & $\mathrm{C} 14$ & 120.0 \\
\hline $\mathrm{O} 1$ & Yb06 Yb07 & $80.1(6)$ & $\mathrm{C} 23$ & $\mathrm{C} 18$ & $\mathrm{C} 17$ & $118(4)$ \\
\hline $\mathrm{O} 1$ & Yb06 C1 & $72.8(13)$ & $\mathrm{C} 23$ & $\mathrm{C} 18$ & C19 & $123(5)$ \\
\hline O39 & Yb06 Yb05 & $142.8(8)$ & C19 & C18 & $\mathrm{C} 17$ & $117(5)$ \\
\hline O39 & Yb06 Yb07 & $143.6(10)$ & $\mathrm{C} 20$ & $\mathrm{C} 21$ & $\mathrm{C} 24$ & $130(6)$ \\
\hline O39 & Yb06 O4 & $137.6(10)$ & $\mathrm{C} 20$ & $\mathrm{C} 21$ & $\mathrm{C} 22$ & $119(5)$ \\
\hline 39 & Yb06 O1 & $136.2(11)$ & $\mathrm{C} 22$ & $\mathrm{C} 21$ & $\mathrm{C} 24$ & $110(5)$ \\
\hline O39 & Yb06 O40 & $55.3(10)$ & $\mathrm{C} 21$ & $\mathrm{C} 20$ & $\mathrm{C} 19$ & $127(6)$ \\
\hline O39 & Yb06 C41 & $27.7(11)$ & $\mathrm{C} 18$ & $\mathrm{C} 23$ & $\mathrm{C} 22$ & $115(5)$ \\
\hline O39 & Yb06 C1 & $88.1(14)$ & $\mathrm{C} 21$ & $\mathrm{C} 22$ & $\mathrm{C} 23$ & $120(5)$ \\
\hline $\mathrm{O} 40$ & Yb06 Yb05 & $100.4(8)$ & $\mathrm{C} 18$ & C19 & $\mathrm{C} 20$ & $114(6)$ \\
\hline $\mathrm{O} 40$ & Yb06 Yb07 & $101.3(7)$ & C9 & O32 & Yb06 & $139(3)$ \\
\hline $\mathrm{O} 40$ & Yb06 O4 & $82.8(9)$ & $\mathrm{C} 46^{7}$ & $\mathrm{C} 48$ & $\mathrm{C} 47$ & $107(5)$ \\
\hline $\mathrm{O} 40$ & Yb06 O1 & $133.4(10)$ & C9 & O31 & Yb07 & $140(4)$ \\
\hline $\mathrm{O} 40$ & Yb06 C41 & $27.9(11)$ & O32 & C9 & $\mathrm{C} 10$ & $123(4)$ \\
\hline
\end{tabular}




\begin{tabular}{|c|c|c|c|c|c|c|}
\hline $\mathrm{O} 40$ & Yb06 C1 & 143.3(13) & $\mathrm{O} 31$ & C9 & $\mathrm{C} 10$ & $118(4)$ \\
\hline O16 & Yb06 Yb05 & $124.0(15)$ & $\mathrm{O} 31$ & C9 & $\mathrm{O} 32$ & $118(4)$ \\
\hline 16 & Yb06 Yb07 & 121.4(9) & C62 & C64 & $\mathrm{C} 63^{11}$ & $122(3)$ \\
\hline $\mathrm{O} 16$ & Yb06 O4 & $148.3(14)$ & C62 & C63 & C64 ${ }^{11}$ & $119(3)$ \\
\hline 016 & Yb06 O1 & $84.2(16)$ & $\mathrm{O} 30$ & C61 & Yb08 & $35(3)$ \\
\hline O16 & Yb06 O39 & $73.5(13)$ & $\mathrm{O} 30$ & C61 & C62 & $102(8)$ \\
\hline O16 & Yb06 O40 & $128.8(13)$ & C62 & C61 & Yb08 & $137(7)$ \\
\hline O16 & Yb06 O37 & $95.5(15)$ & O29 & C61 & Yb08 & $101(5)$ \\
\hline $\mathrm{O} 16$ & Yb06 C41 & 101.1(15) & $\mathrm{O} 29$ & C61 & $\mathrm{O} 30$ & $134(8)$ \\
\hline O16 & Yb06 C1 & $14.6(15)$ & O29 & C61 & C62 & $121(9)$ \\
\hline 016 & Yb06 O32 & $93.8(18)$ & C61 & $\mathrm{O} 30$ & Yb08 & $123(6)$ \\
\hline O16 & Yb06 O3 & $82.3(11)$ & C64 & C62 & C61 & $126(6)$ \\
\hline $\mathrm{O} 37$ & Yb06 Yb05 & $72.7(11)$ & C63 & C62 & C64 & $119(3)$ \\
\hline $\mathrm{O} 37$ & Yb06 Yb07 & $130.9(10)$ & C63 & C62 & C61 & $112(6)$ \\
\hline $\mathrm{O} 37$ & Yb06 O4 & $90.5(11)$ & C61 & O29 & Yb07 & $135(6)$ \\
\hline $\mathrm{O} 37$ & Yb06 O1 & 72.1(14) & Yb07 & $\mathrm{O} 3$ & Yb06 & $101.6(9)$ \\
\hline $\mathrm{O} 37$ & Yb06 O39 & 73.1(15) & Yb08 & $\mathrm{O} 3$ & Yb06 & $107.0(8)$ \\
\hline O37 & Yb06 O40 & $72.7(14)$ & Yb08 & $\mathrm{O} 3$ & Yb07 & $105.8(10)$ \\
\hline 0 & Yb06 C41 & $67.3(17)$ & & & & \\
\hline
\end{tabular}

Symmetry transformations used to generate equivalent atoms:

${ }^{1} 1-\mathrm{X}, 1-\mathrm{Y},-\mathrm{Z} ;{ }^{2} 3 / 2-\mathrm{X}, 1 / 2-\mathrm{Y}, 1-\mathrm{Z} ;{ }^{3} 1-\mathrm{X},+\mathrm{Y}, 1 / 2-\mathrm{Z} ;{ }^{4} 3 / 2-\mathrm{X},-1 / 2+\mathrm{Y}, 1 / 2-\mathrm{Z} ;{ }^{5} 1 / 2+\mathrm{X}, 1 / 2-\mathrm{Y}, 1 / 2+Z ;{ }^{6} 3 / 2-\mathrm{X}, 1 / 2+\mathrm{Y}, 1 / 2-\mathrm{Z}$; ${ }^{7} 3 / 2-\mathrm{X}, 1 / 2-\mathrm{Y},-\mathrm{Z} ;{ }^{8}-1 / 2+\mathrm{X}, 1 / 2-\mathrm{Y},-1 / 2+\mathrm{Z} ;{ }^{9}+\mathrm{X}, 1-\mathrm{Y},-1 / 2+\mathrm{Z} ;{ }^{10}+\mathrm{X}, 1-\mathrm{Y}, 1 / 2+\mathrm{Z} ;{ }^{11} 2-\mathrm{X}, 1-\mathrm{Y}, 1-\mathrm{Z}$ 
Table S7: Hydrogen Atom Coordinates $\left(\AA \times 10^{4}\right)$ and Isotropic Displacement Parameters $\left(\AA^{2} \times 10^{3}\right)$ for MOF1140(Yb).

\begin{tabular}{|c|c|c|c|c|}
\hline Atom & $x$ & $y$ & $z$ & $\mathrm{U}(\mathrm{eq})$ \\
\hline H35 & 4142 & 2854 & -302 & 125 \\
\hline H55 & 5349 & 2338 & 2516 & 136 \\
\hline H31 & 6274 & 1614 & 831 & 127 \\
\hline H51 & 7310 & 2107 & 4486 & 104 \\
\hline H47 & 7867 & 2801 & 828 & 223 \\
\hline H52 & 7559 & 2136 & 5517 & 127 \\
\hline H38 & 3700 & 2178 & -1688 & 130 \\
\hline H59 & 5433 & 5356 & 56 & 219 \\
\hline $\mathrm{H} 30$ & 6535 & 1193 & 986 & 137 \\
\hline H39 & 4575 & 2293 & -865 & 108 \\
\hline $\mathrm{H} 4$ & 6188 & 3796 & 2003 & 228 \\
\hline $\mathrm{H} 7$ & 7670 & 4119 & 2452 & 236 \\
\hline H56 & 5173 & 3081 & 2175 & 133 \\
\hline H6 & 7426 & 3720 & 2116 & 246 \\
\hline H60 & 5240 & 4842 & 1002 & 230 \\
\hline $\mathrm{H} 43$ & 5357 & 5365 & 2458 & 171 \\
\hline H36 & 3278 & 2721 & -1071 & 132 \\
\hline H15 & 6876 & 4730 & 4314 & 252 \\
\hline H11 & 8065 & 5212 & 5378 & 253 \\
\hline $\mathrm{H} 12$ & 8134 & 5107 & 6278 & 247 \\
\hline $\mathrm{H} 20$ & 3927 & 3925 & -164 & 177 \\
\hline $\mathrm{H} 23$ & 4906 & 3571 & -644 & 160 \\
\hline $\mathrm{H} 22$ & 4292 & 3923 & -1259 & 166 \\
\hline H19 & 4428 & 3552 & 411 & 197 \\
\hline $\mathrm{H} 48$ & 8254 & 2736 & 239 & 256 \\
\hline H64 & 9547 & 4788 & 4037 & 294 \\
\hline H63 & 9580 & 5287 & 5200 & 251 \\
\hline H6A & 7642 & 1120 & 3196 & 180 \\
\hline H6B & 7591 & 1375 & 3403 & 180 \\
\hline H5A & 7006 & 1850 & 2962 & 197 \\
\hline H5B & 7294 & 1610 & 3305 & 197 \\
\hline H3A & 6366 & 4350 & 2472 & 499 \\
\hline H3B & 6079 & 4102 & 2239 & 499 \\
\hline $\mathrm{H} 2 \mathrm{~A}$ & 6617 & 4467 & 4766 & 452 \\
\hline $\mathrm{H} 2 \mathrm{~B}$ & 6825 & 4487 & 5453 & 452 \\
\hline
\end{tabular}




$\begin{array}{lllll}\text { H1A } & 5646 & 4548 & 2473 & 192 \\ \text { H1B } & 5207 & 4423 & 2400 & 192\end{array}$

Table S8: Atomic Occupancy for MOF-1140(Yb).

\begin{tabular}{llllll}
\hline Atom Occupancy & Atom Occupancy & Atom Occupancy \\
\hline N6 & $0.51(5)$ & H6A & $0.51(5)$ & H6B & $0.51(5)$ \\
N5 & $0.49(5)$ & H5A & $0.49(5)$ & H5B & $0.49(5)$ \\
N1 & 0.5 & H1A & 0.5 & H1B & 0.5
\end{tabular}




\section{Post Synthetic Modification and Characterization}

As-synthesized crystals of MOF-1114(Yb) or MOF-1140(Yb) ( 50 mg) were transferred to $1.5 \mathrm{~mL}$ centrifuge tubes and washed with DMF ( $3 \mathrm{x})$ followed by acetonitrile $(3 \mathrm{x})$. Crystals were then transferred to 4 dram glass vials along with about $250 \mu \mathrm{L}$ of acetonitrile and $1 \mathrm{~mL}$ of methyl propiolate. Vials were then left to react for $\sim 24$ hours in a preheated $75^{\circ} \mathrm{C}$ oven or at room temperature. After 24 hours, modified crystals were washed with acetonitrile (5x) and dried under reduced pressure.

Powder X-ray Diffraction Analysis:

Powder X-ray diffraction patterns were obtained to confirm that crystallinity was maintained after post synthetic modification of MOF crystals.

MOF-1114(Yb)

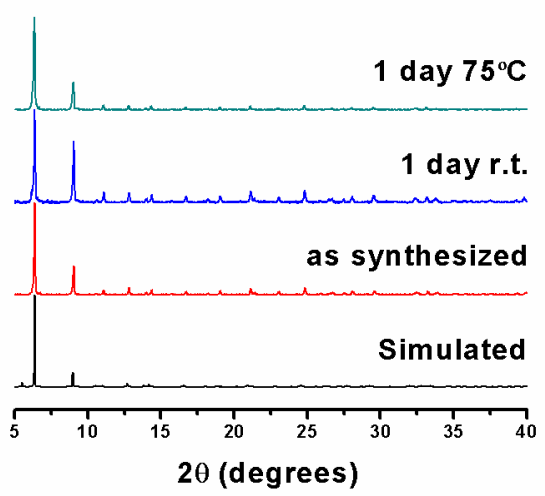

MOF-1140(Yb)

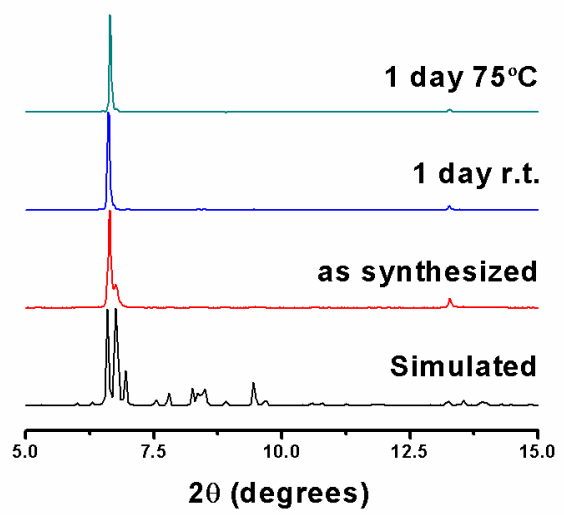

Figure S4: PXRD patterns for as-synthesized crystals (red) and after soaking in MP for 16 hours at r.t. (blue) and at $75^{\circ} \mathrm{C}$ (cyan). All MOF crystals were washed with DMF prior to collecting PXRD patterns. Experimental powder patterns for MOF-1140(Yb) exhibit 001 preferred orientation expected for plate-like crystals (see Fig. S2). The patterns simulated from single crystal data are shown in black. 


\section{LCMS Analysis:}

LCMS analyses were performed on a Thermo Scientific Q-Exactive Oribtrap mass spectrometer with a Thermo Scientific Hypersil GOLD C $\mathrm{C}_{18}$ Selectivity LC column $(1.9 \mu \mathrm{m}, 10$ cm x 3mm, catalogue \# 25002-103030). Samples were run for $30 \mathrm{~min}$. ESI negative mode with $\mathrm{H}_{2} \mathrm{O}$ :acetonitrile 95:5 to 5:95 linear solvent gradient at constant flow rate of $3.00 \mu \mathrm{L} / \mathrm{min}$. MOF crystals $(\sim 10 \mathrm{mg})$ were dissolved in $0.5 \mathrm{~mL}$ DMSO and $20 \mu \mathrm{L}$ conc. $\mathrm{H}_{2} \mathrm{SO}_{4}$ and then diluted 100x with acetonitrile. A representative chromatogram for modified MOF-1114(Yb) (soaked in MP $75^{\circ} \mathrm{C}, 16 \mathrm{hrs}$ ) is shown in Fig. S5 and the corresponding mass spectra showing elemental composition with isotopic resolution for each of the expected products shown in Fig. S6-S10. Chromatogram peak at 6.2 minutes with base peak of $180.03 \mathrm{~m} / \mathrm{z}$ corresponds to unreacted $\mathrm{NH}_{2-}$ BDC. The chromatograms for each of the modified MOFs at different temperatures are then shown in Fig. S11. 


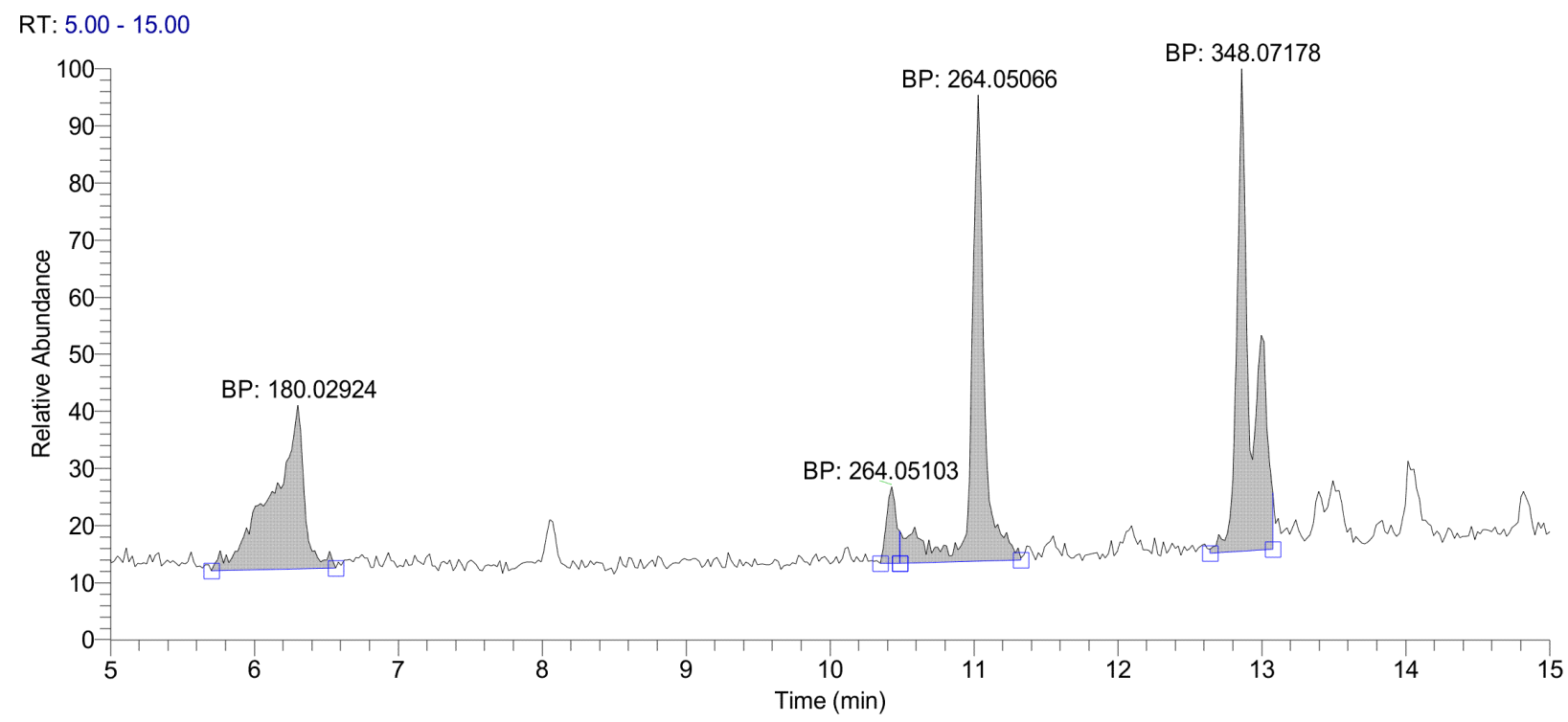

Figure S5: Chromatogram of modified MOF-1114 for 5-15 minute retention time. Relevant peaks corresponding to major linker-modification products are shaded.

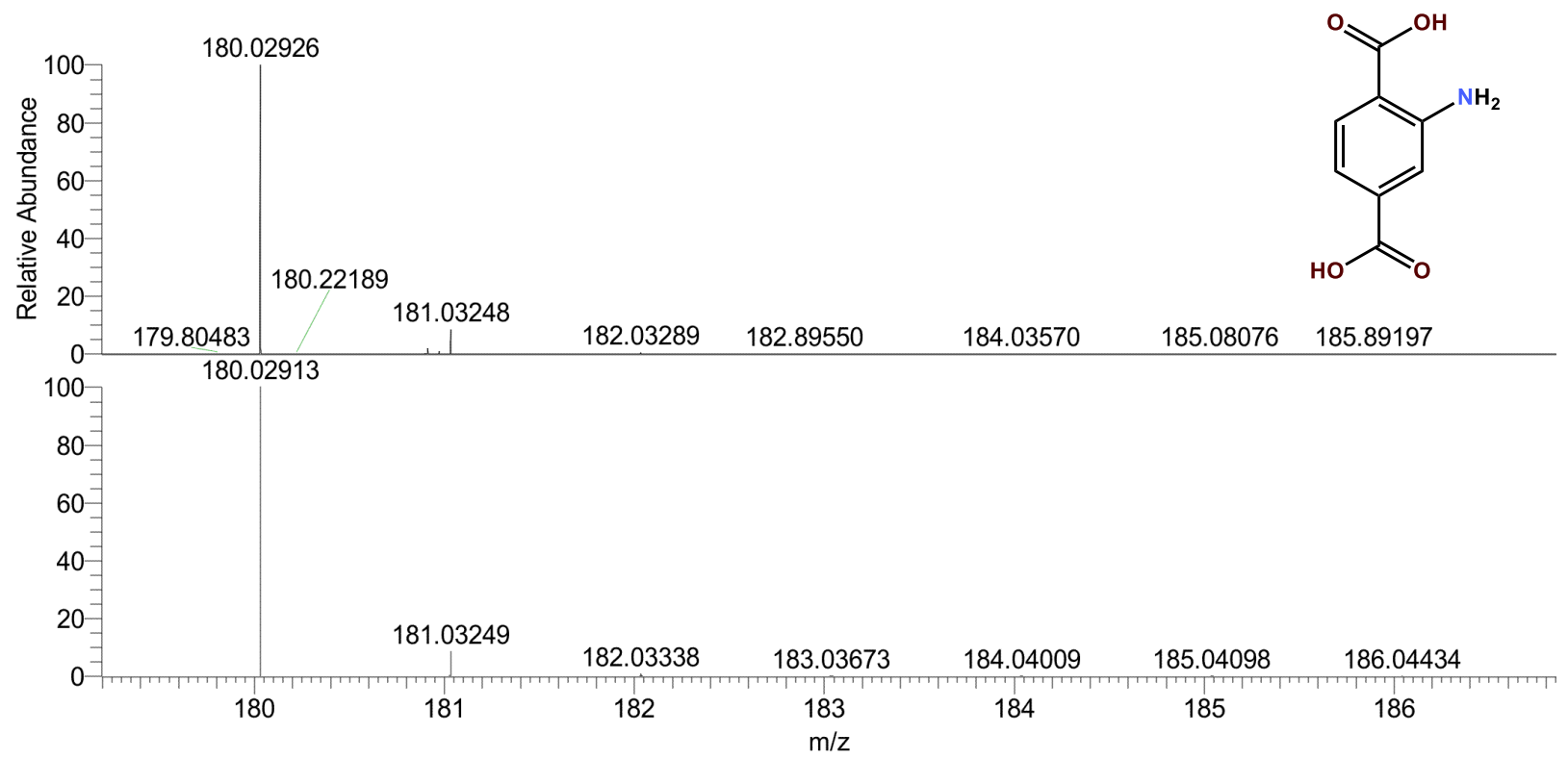

Figure S6: Mass spectrum of modified MOF-1114 for 5.93-6.38 retention time. The peak at $180.029 \mathrm{~m} / \mathrm{z}$ is assigned to unreacted linker, $\mathrm{NH}_{2}-\mathrm{BDC}$. 


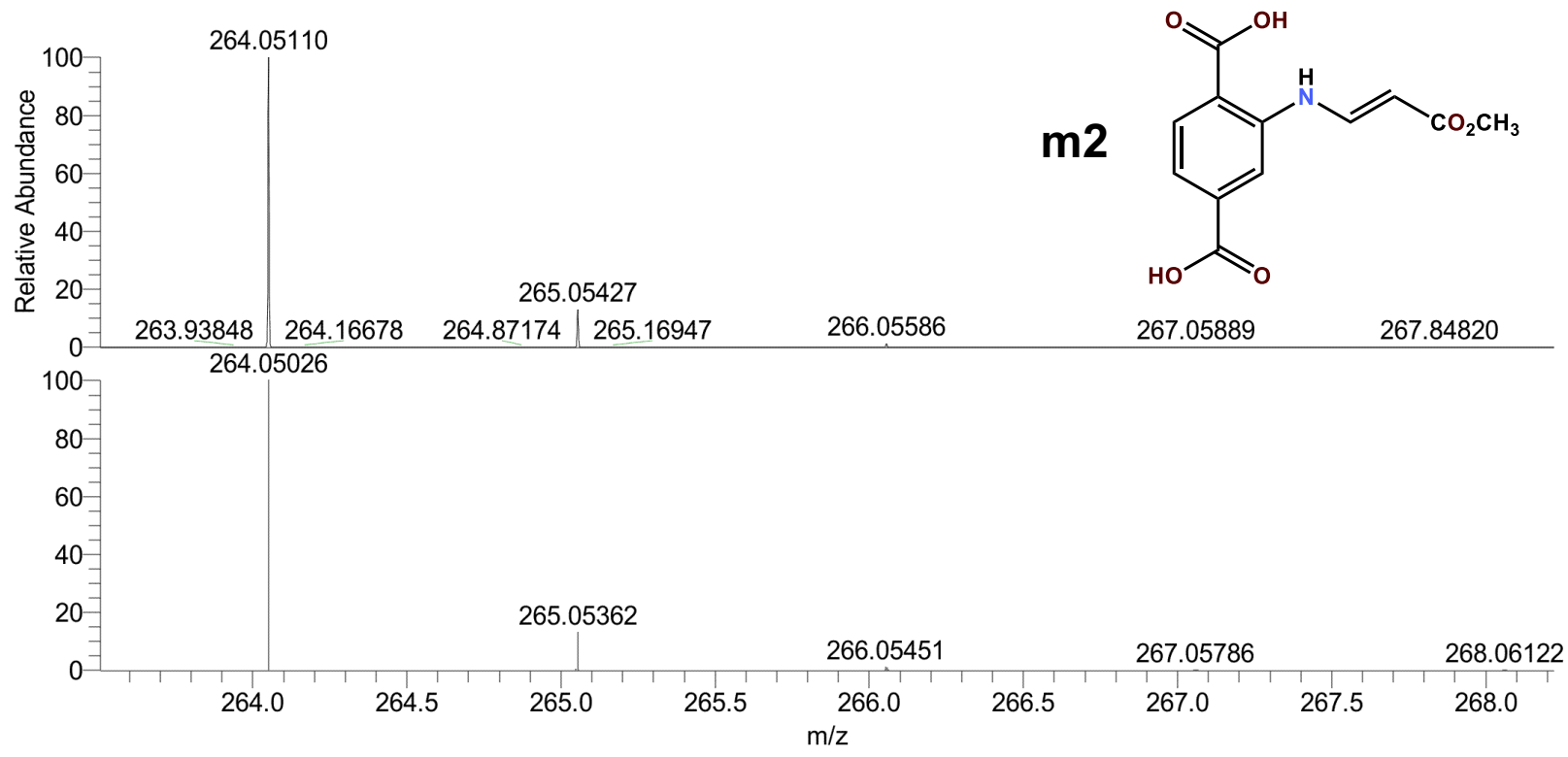

Figure S7: Mass spectrum of modified MOF-1114 for 10.39-10.47 retention time. This first peak at 265.05 $\mathrm{m} / \mathrm{z}$ is assigned to the E-isomer of the monoadduct, $\mathbf{m} \mathbf{2}$.

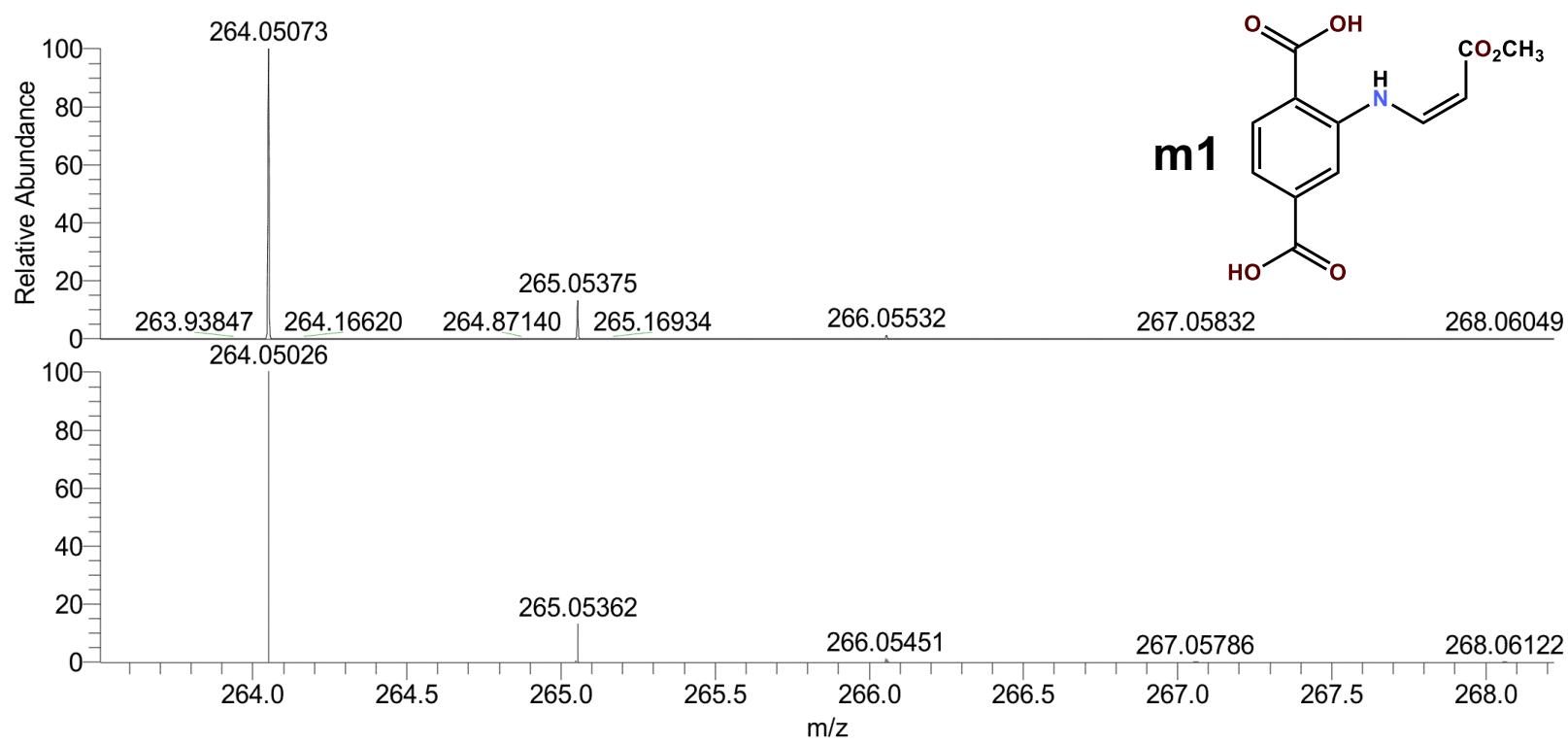

Figure S8: Mass spectrum of modified MOF-1114 for 10.99-11.09 retention time. This second peak at $265.05 \mathrm{~m} / \mathrm{z}$ is assigned to the Z-isomer of the monoadduct, $\mathbf{m} \mathbf{1}$. 


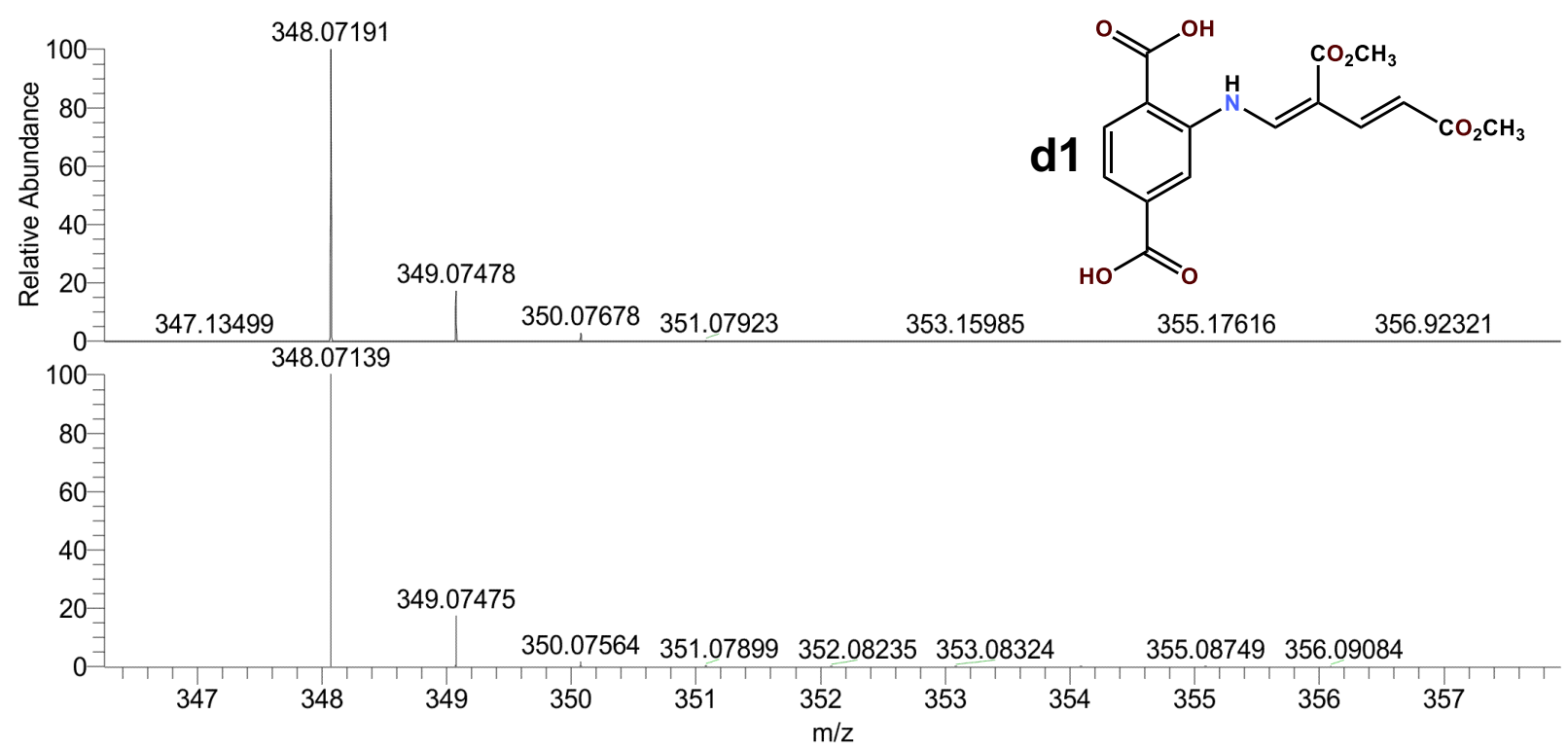

Figure S9: Mass spectrum of modified MOF-1114 for 12.84-12.88 retention time. This first peak at 348.07 $\mathrm{m} / \mathrm{z}$ is assigned to the (Z,E)-isomer of the diadducts, $\mathbf{d} 1$.

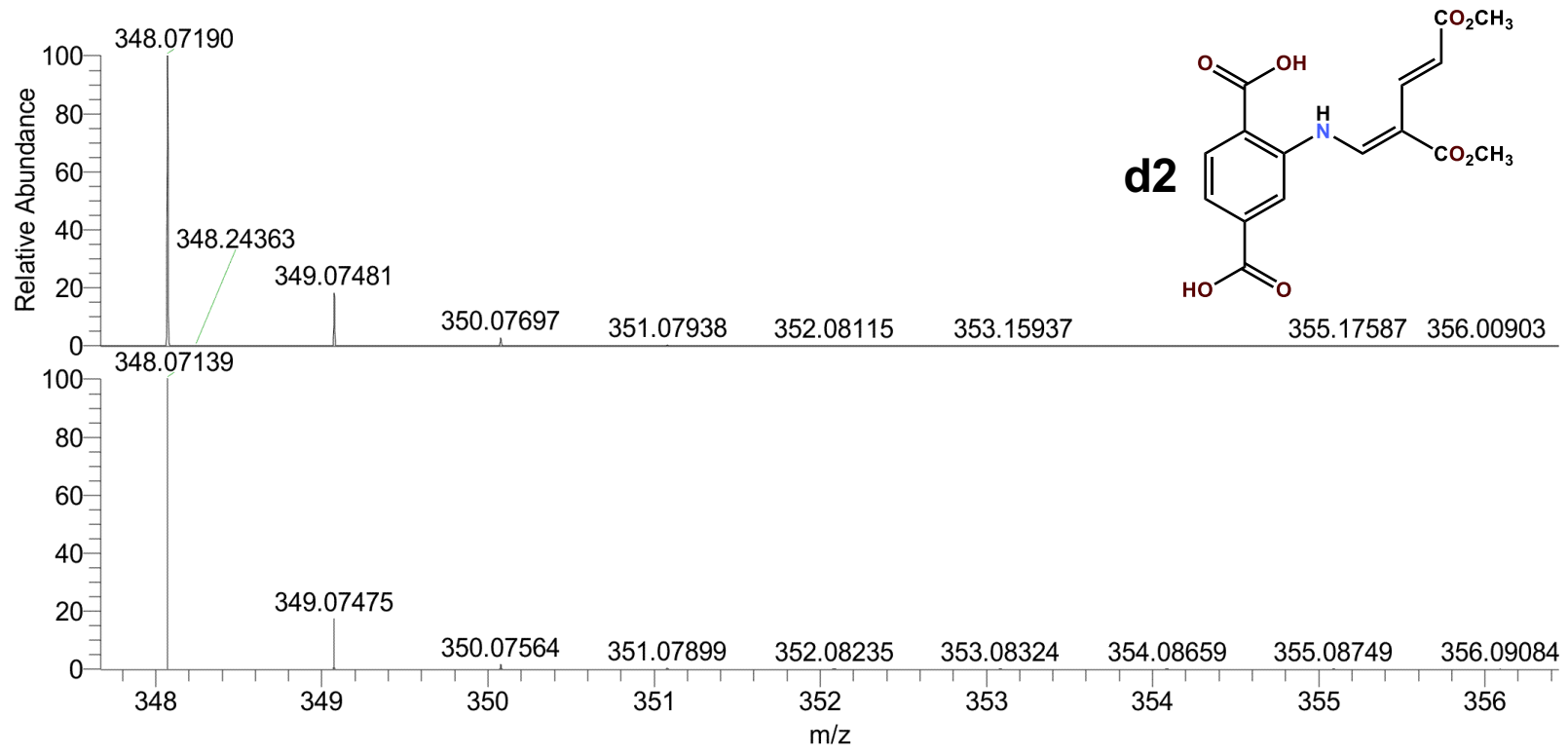

Figure S10: Mass spectrum of modified MOF-1114 for 12.96-13.02 retention time. This second peak at $348.07 \mathrm{~m} / \mathrm{z}$ is assigned to the (E,E)-isomer of the diadducts, $\mathbf{d} 2$. 


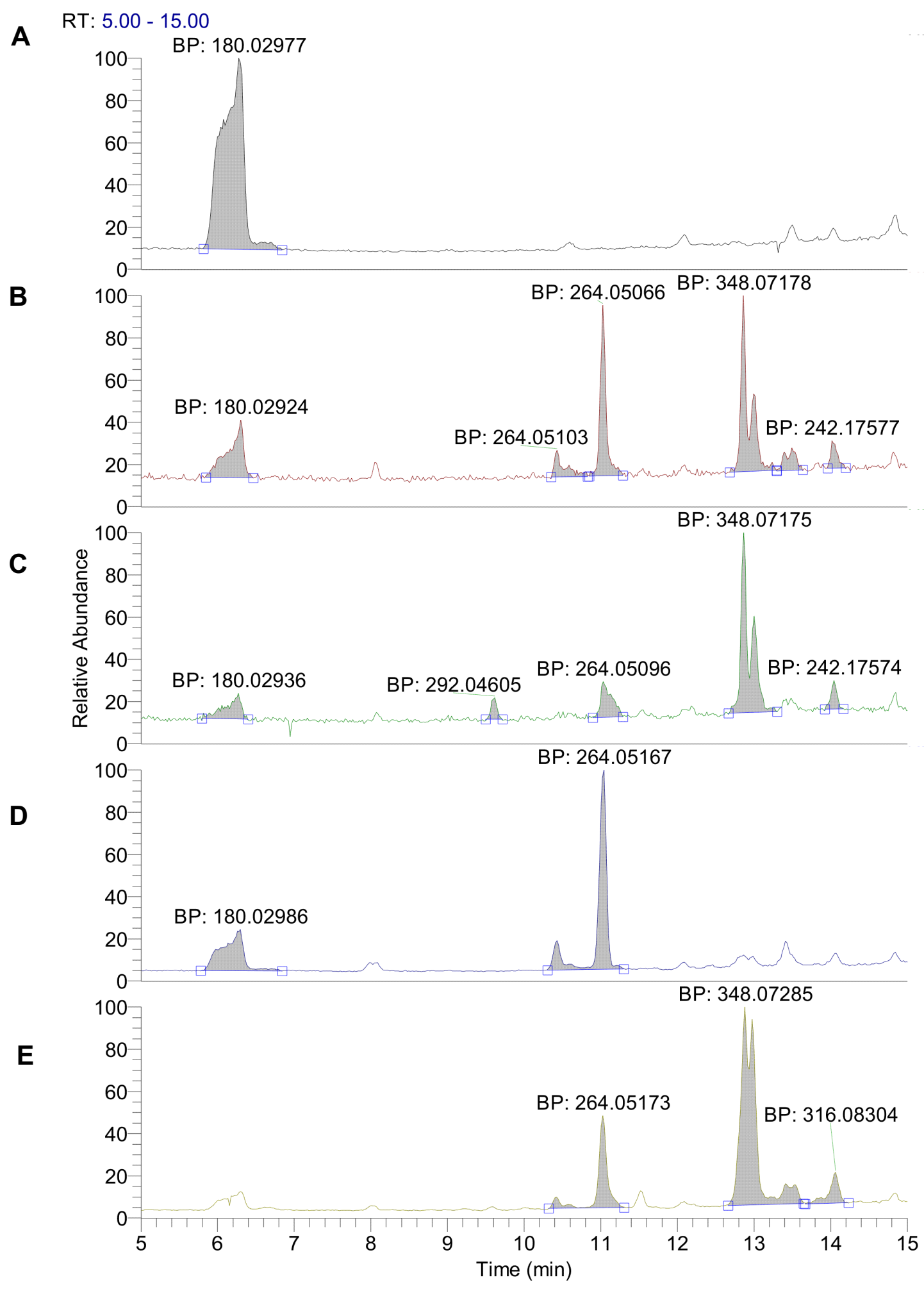

Figure S11: LCMS Chromatograms for MOF post synthetic modification reactions which qualitatively show shifting product distributions; A) Unmodified MOF-1114(Yb); B) and C) MOF-1114(Yb) modified at r.t. and at $75^{\circ} \mathrm{C}$, respectively; D) and E) MOF-1140(Yb) modified at r.t and at $75^{\circ} \mathrm{C}$, respectively. 
Product Separation and NMR Analysis of Modified Linkers:

Approximately $50 \mathrm{mg}$ of modified crystals of MOF-1114(Yb) (soaked in MP $75^{\circ} \mathrm{C}, 16 \mathrm{hrs}$ ) were dissolved in $0.5 \mathrm{~mL}$ DMSO, $0.5 \mathrm{~mL}$ acetonitrile, and $20 \mu \mathrm{L}$ conc. $\mathrm{H}_{2} \mathrm{SO}_{4}$ for prep-scale partial separation by reverse phase HPLC. Separation was performed at ambient temperature with an Agilent 1200 liquid chromatographic system equipped with diode array and multiple wavelength detectors using an Ascentis $\mathrm{C}_{18}$ column $(5 \mu \mathrm{m}, 25 \mathrm{~cm}$ x $4.6 \mathrm{~mm}$, catalogue \# 581325U). A 50-minute run with a flow rate of $1 \mathrm{~mL} / \mathrm{min}$ with the linear solvent gradient tabulated below.

\begin{tabular}{|c|c|c|}
\hline Minute & $\mathrm{H}_{2} \mathrm{O}(\%)$ & Acetonitrile (\%) \\
\hline 0 & 95 & 5 \\
\hline 5 & 95 & 5 \\
\hline 10 & 70 & 30 \\
\hline 30 & 50 & 50 \\
\hline 45 & 50 & 50 \\
\hline 46 & 0 & 100 \\
\hline 49 & 0 & 100 \\
\hline 50 & 95 & 5 \\
\hline
\end{tabular}

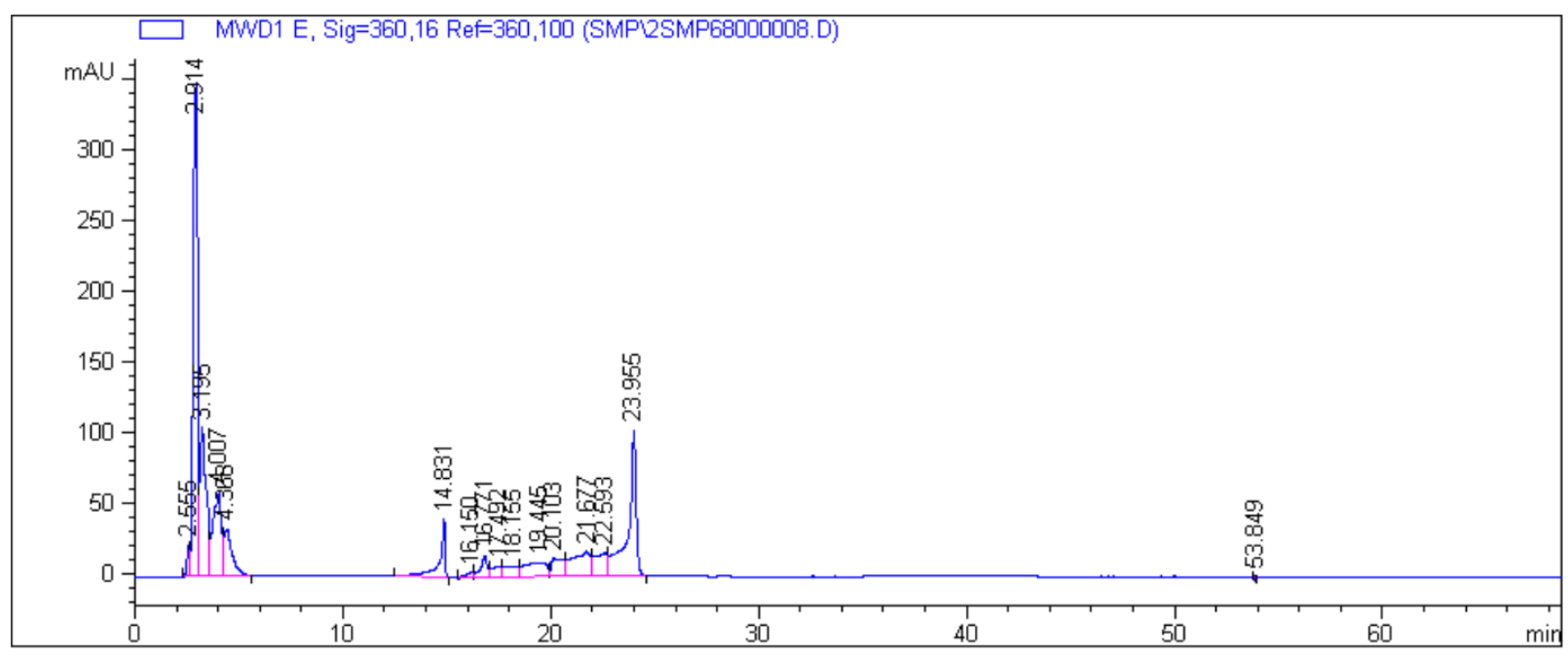

Figure S12: HPLC separation method and corresponding chromatogram for separation of modified $\left(75^{\circ} \mathrm{C}, 16 \mathrm{hrs}\right)$ and dissolved MOF-1114(Yb), monitored via absorbance at $360 \mathrm{~nm}$. 
Three fractions were collected and lyophilized for ${ }^{1} \mathrm{H}$ NMR analysis for the followed retention times: 18.2-20.1 minutes, 20.1-22.6 minutes, and 22.6-24 minutes (Fig. S13).

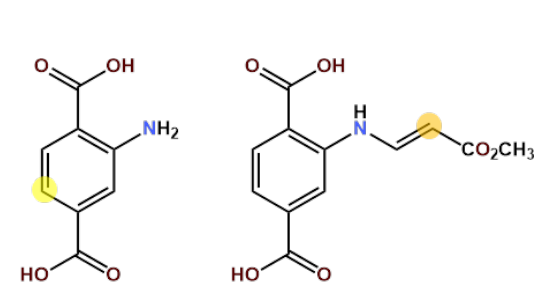

$\mathrm{NH}_{2}-\mathrm{BDC}$

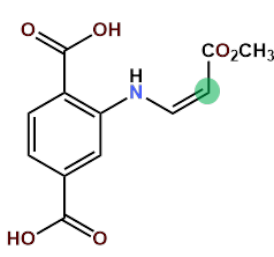

m1

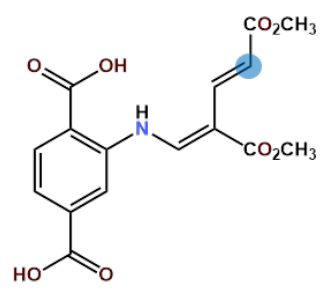

d2

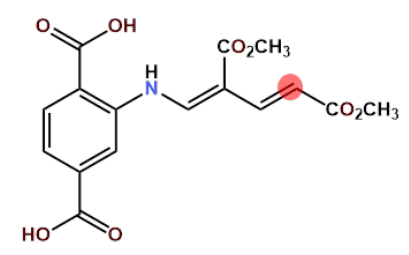

d1
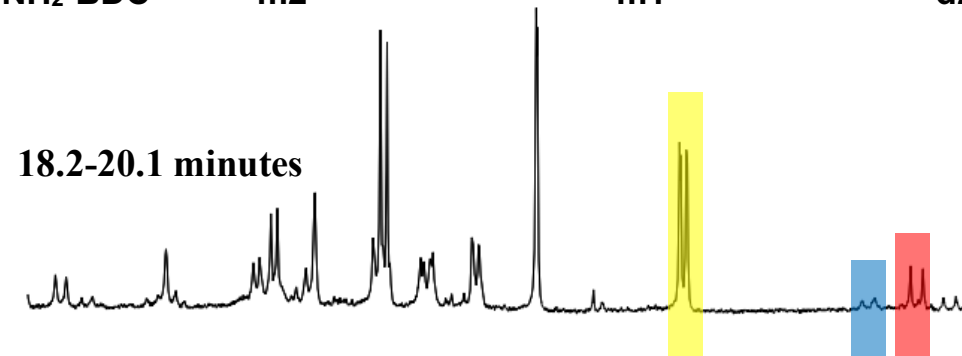

20.1-22.6 minutes

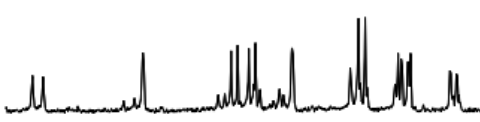

22.6-24.0 minutes
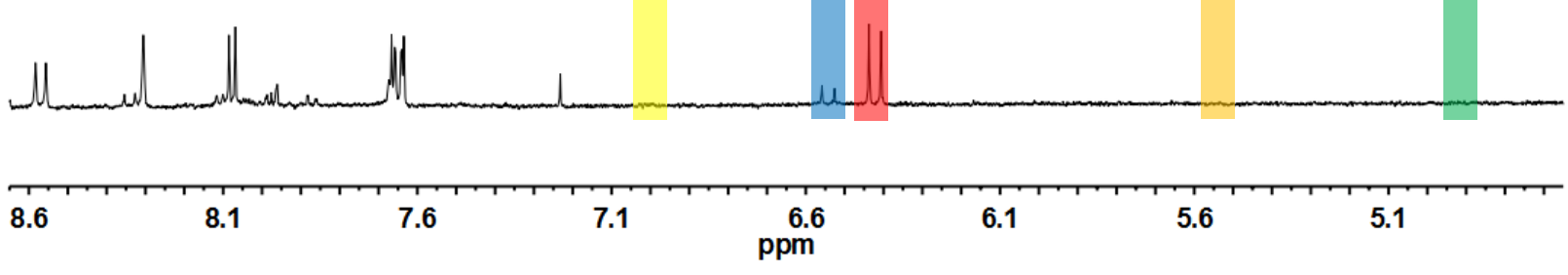

Figure S13: ${ }^{1} \mathrm{H}$ NMR spectra (DMSO-d6) of HPLC-separated fractions (Fig. S12) of linker modification products. Select protons on individual species highlighted and color-coded to match well resolved peaks on spectra. 
From comparative chemical shifts, it was inferred that the doublets occurring in the wellresolved region between 4.8 and 6.6 ppm correspond to the vinylogous protons on the modified linker species. The disappearance of 4.9 and 5.5 doublets in later fractions is consistent with the monoadducts which elute sooner based on LCMS (Fig. S5-S11) and the diadducts should elute last with corresponding doublets at 6.4 and $6.5 \mathrm{ppm}$. Coupling constants were used to differentiate between $\mathrm{E} / \mathrm{Z}$ isomers (protons that are trans across 1,2-disubstituted olefins have a larger coupling constant than those that are cis): (500 MHz DMSO) $\mathbf{m 1} \delta 4.89$ (d, J=10); m2 $\delta$ $5.55(\mathrm{~d}, \mathrm{~J}=15) ; \mathbf{d} 1 \delta 6.41(\mathrm{~d}, \mathrm{~J}=15) ; \mathbf{d} 2 \delta 6.53(\mathrm{~d}, \mathrm{~J}=10)$. For product ratio determination, modified MOF crystals of MOF-1114 and MOF-1140 were dissolved in 0.5 mL DMSO-d6 and $20 \mu \mathrm{L} \mathrm{D}_{2} \mathrm{SO}_{4}$ and the above-mentioned peaks were integrated. Results summarized in Figure 3.

Compound d1 (below) was eventually isolated via HPLC from fractions collected from 2426 minute retention times using the solvent gradient shown in Fig. S12 : (500 MHz DMSO) $\delta$ $8.56(\mathrm{~d}, \mathrm{~J}=13 \mathrm{~Hz}, 1 \mathrm{H}), 8.30$ (d, J=1.25 Hz, 1H), 8.08 (d, J=8.5 Hz, 1H), 7.65 (d, J=15.5 Hz, 1H), $7.64(\mathrm{dd}, \mathrm{J}=8.25, \mathrm{~J}=1.25 \mathrm{~Hz}, 1 \mathrm{H}), 6.42(\mathrm{~d}, \mathrm{~J}=16 \mathrm{~Hz}, 1 \mathrm{H}), 3.83(\mathrm{~s}, 3 \mathrm{H}), 3.65$ (s, 3H).

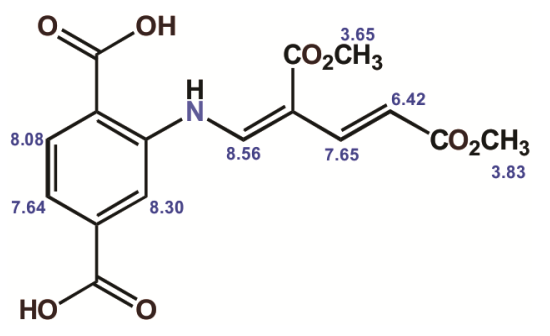




\section{Spectral Data and Imaging}

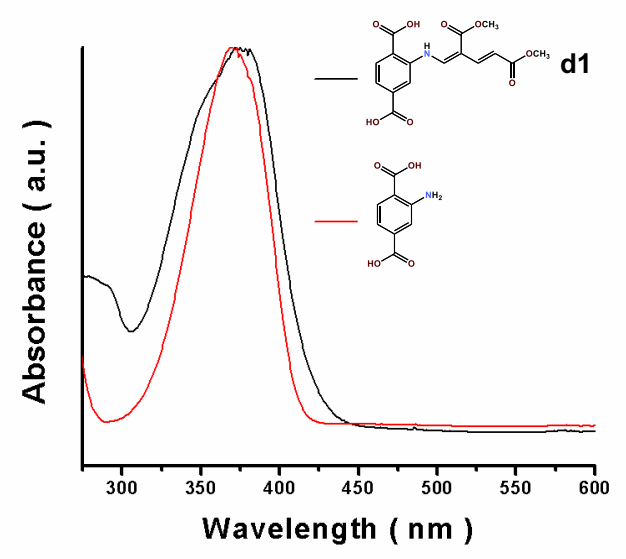

Figure S14: UV-vis spectra of purified d1 (black) and $\mathrm{NH}_{2}-\mathrm{BDC}$ (red) dissolved in DMF.

Optical Microscopy Imaging:

Epifluorescence microscopy images were acquired with an inverted Nikon Eclipse Ti microscope equipped with an EMCCD Evolve camera from Photometrics. The Nikon Intensilight C-HGFIE mercury-halide lamp was used as excitation source.

For imaging MOF crystals (Fig. S15 and S18), the $\mathrm{Yb}^{3+}$ signal was observed using the following set of filters : a $482 \mathrm{~nm}$ band pass $35 \mathrm{~nm}$ excitation filter, a $801 \mathrm{~nm}$ dichroic beam splitter, and a $996 \mathrm{~nm}$ band pass $70 \mathrm{~nm}$ emission filter. Fluorescent images were obtained with Nikon Plan Apo 20x and Apo TIRF 60x objective after 5 seconds of exposure time.

For imaging of miniaturized MOF fragments in water with $0.5 \%$ Tween-20 in the presence of RAW 264.7 cells (Fig. S20), the $\mathrm{Yb}^{3+}$ signal was observed using the following set of filters: a $482 \mathrm{~nm}$ band pass $35 \mathrm{~nm}$ excitation filter, a $801 \mathrm{~nm}$ dichroic beam splitter, and a long pass 785 $\mathrm{nm}$ emission filter. Fluorescent images were obtained with a Nikon Plan Fluor 40x objective after 2 seconds of exposure time. An Okolab incubation chamber adapted to the microscope allowed to maintain constant for cells the incubation parameters at $37^{\circ} \mathrm{C}$ with a humidified $95 \%$ 
air/5\% $\mathrm{CO}_{2}$ atmosphere. All microscopy images were acquired and processed with Nikon NIS Elements AR and ImageJ software.
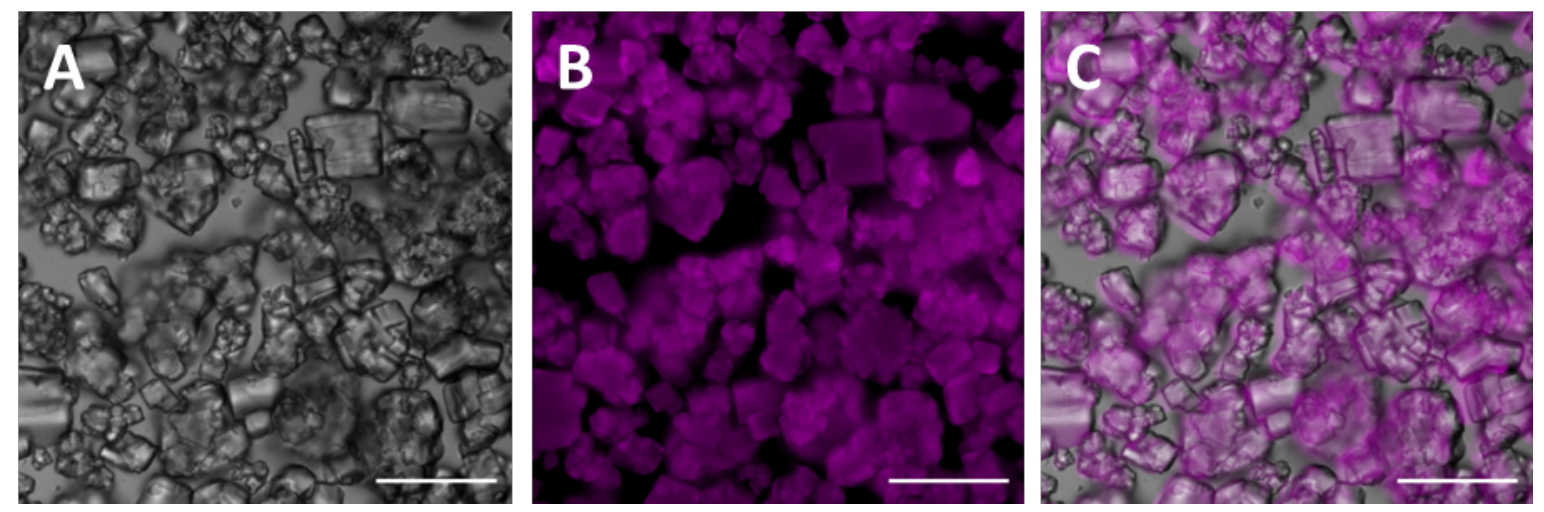

Figure S15: Microscopy images of bulk MOF in DMF. Brightfield imaging (A), NIR epifluorescence imaging (B), and merge (C). $\lambda_{\text {ex }}$ : $482 \mathrm{~nm}$ band pass $35 \mathrm{~nm}, \lambda_{\mathrm{em}}: 996 \mathrm{~nm}$ band pass $70 \mathrm{~nm}, \tau_{\text {exp }}: 5 \mathrm{~s}$. Objective $20 \times$. Scale bar $=100 \mu \mathrm{m}$. 
Miniaturization and Size Distribution:

Crystals of MOF-1114 in DMF were grinded with mortar and pestle and subjected to ultrasonication/probe sonication to obtain small MOF fragments. These fragments were subsequently post synthetically modified with methyl propiolate following the procedure described in Section 3, Post Synthetic Modification and Characterization.

The size distribution of the as-synthesized bulk MOF (MOF-1114(Yb)), the miniaturized MOF in water, and the miniaturized MOF in water with $0.5 \%$ Tween- 20 was determined by a microscopy-based method using brightfield image acquired with an inverted Nikon Eclipse Ti microscope equipped with an EMCCD Evolve camera from Photometrics. Twenty-five fields of view for each of the 3 MOF samples were acquired with a Nikon Apo TIRF 60x objective and analyzed by ImageJ software using the "analyze particles" plug-in on previously binarized and thresholded pictures. The size distribution was fitted by a Gaussian curve with OriginPro8 software (Fig. S16). The mean particle size and the polydispersity were determined from this fit. Polydispersity $(\%)=$ standard deviation/average particles size $\times 100$. 

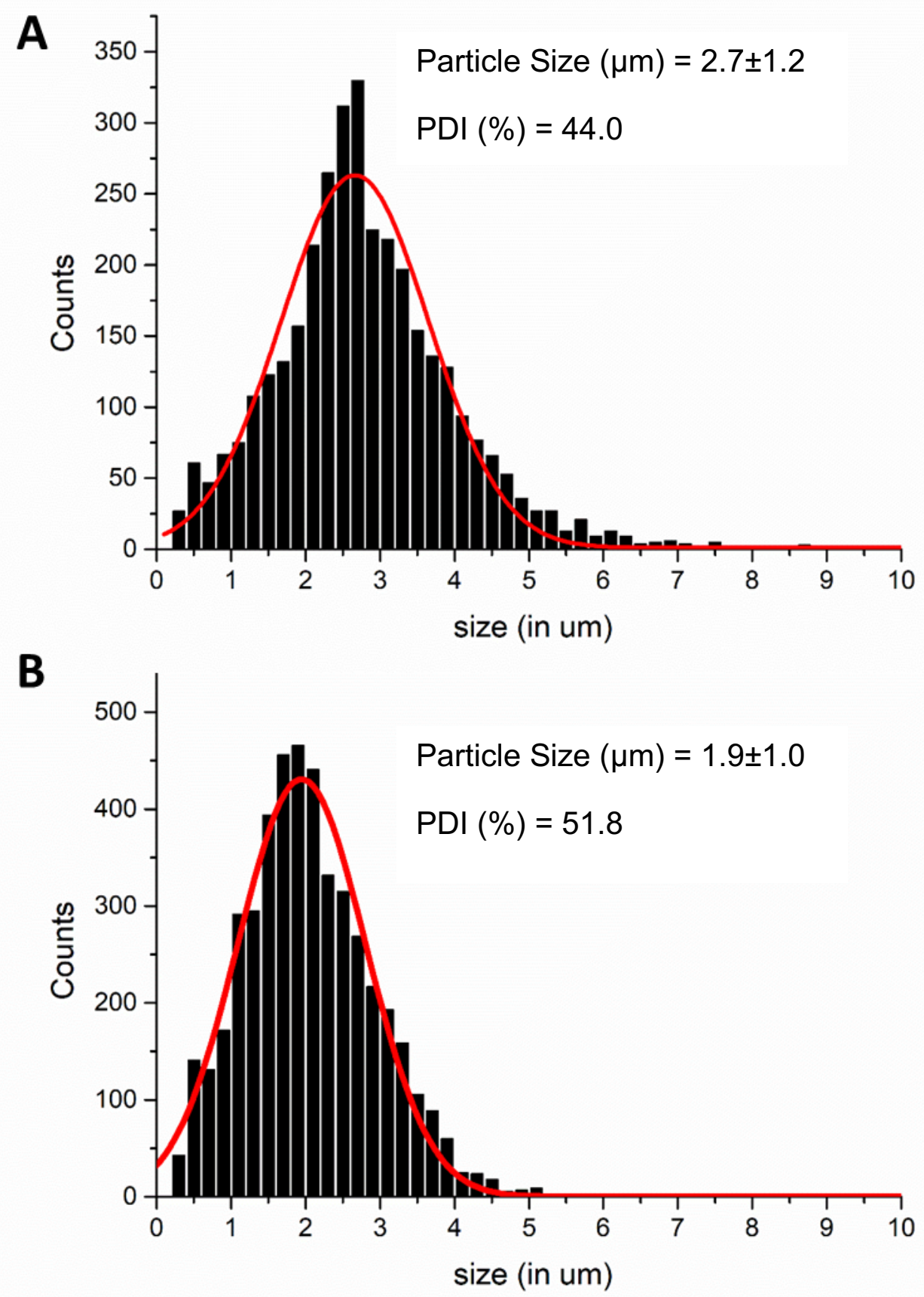

Figure S16: Size distribution histogram (black) obtained from brightfield images analysis and Gaussian fitting (red) of miniaturized MOF-1114(Yb) in water (A), and in water with 0.5\% Tween-20 (B). 


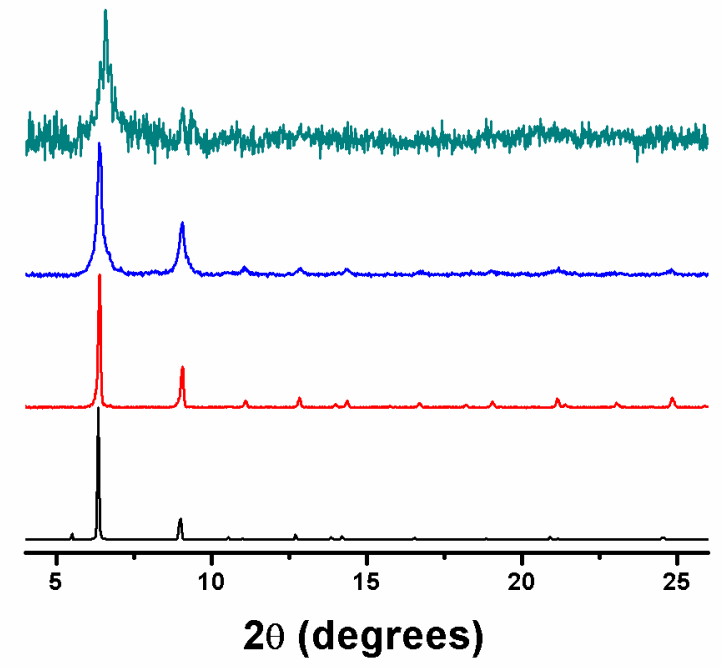

Figure S17: PXRD patterns for MOF-1114(Yb) fragments. Experimental PXRD pattern for as-synthesized MOF-1114(Yb) in DMF (red), PXRD pattern simulated from single crystal data (black), modified MOF fragments after 1 day in water (blue), and unmodified MOF after 1 day in water (teal). 

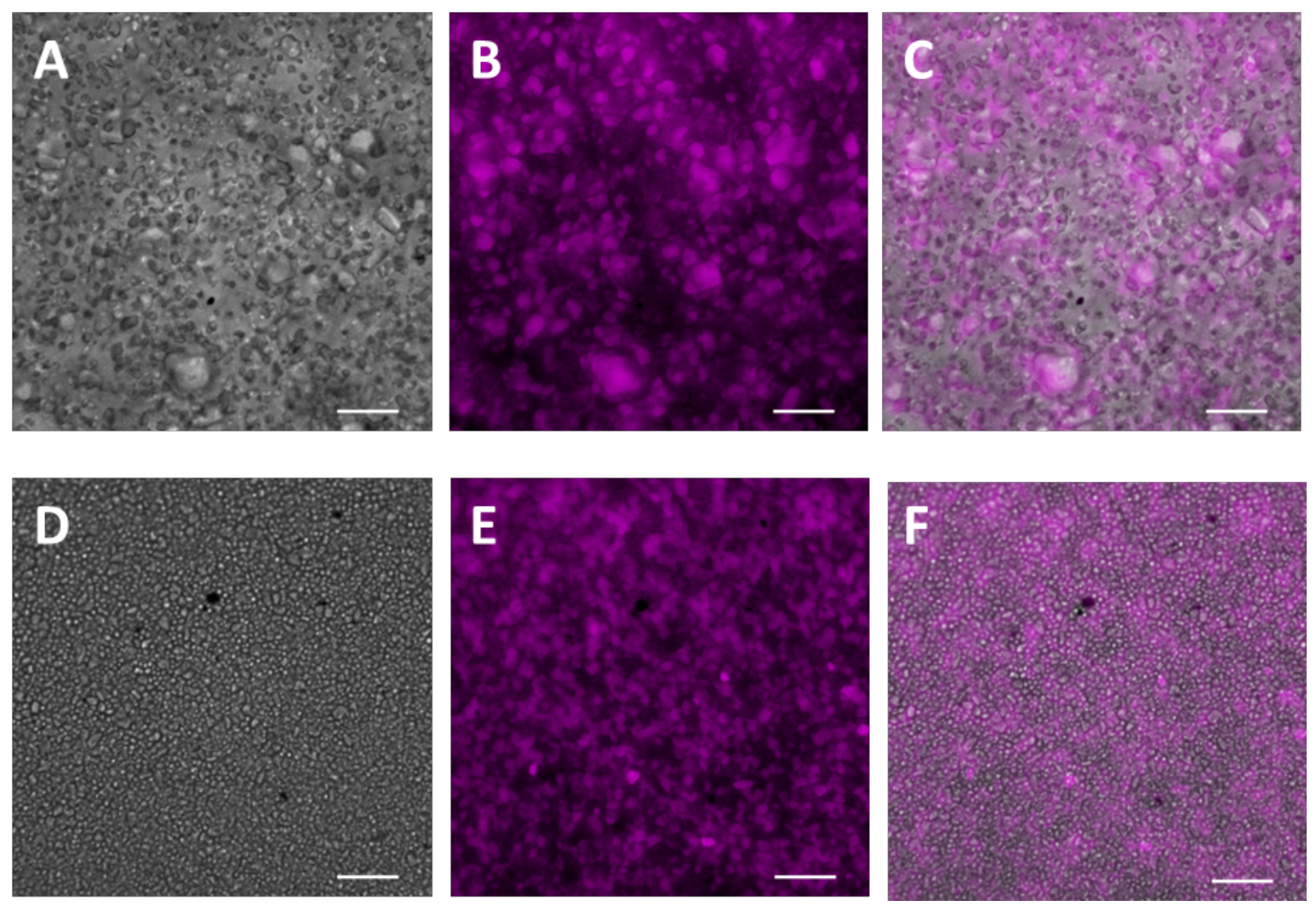

Figure S18: Microscopy images of miniaturized MOF in water (A, B, C), and in water with $0.5 \%$ Tween20 (D, E, F). Brightfield imaging (A, D), NIR epifluorescence imaging (B, E), and merge (C, F). $\lambda_{\text {ex }}: 482$ $\mathrm{nm}$ band pass $35 \mathrm{~nm}, \lambda_{\mathrm{em}}: 996 \mathrm{~nm}$ band pass $70 \mathrm{~nm}, \tau_{\text {exp }}: 5 \mathrm{~s}$. Objective $60 \times$. Scale bar $=20 \mu \mathrm{m}$. 
A

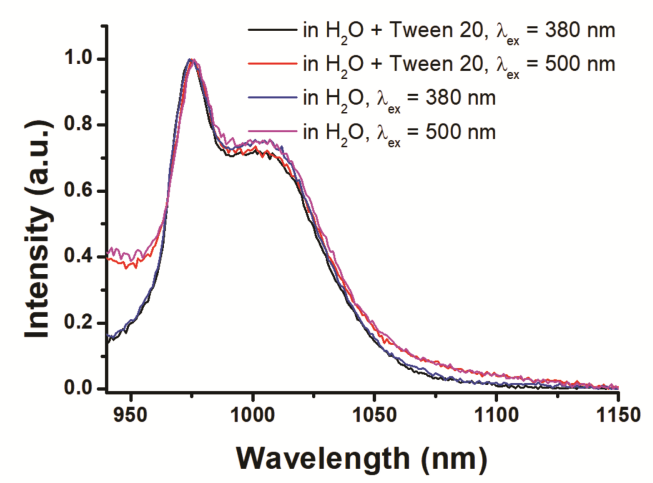

B

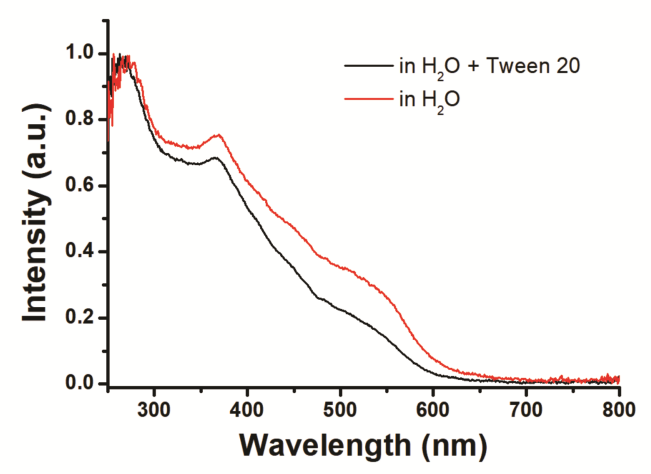

C

\begin{tabular}{cccccc}
\hline \multirow{2}{*}{ MOF } & Sample & $\mathbf{T}_{1} / \boldsymbol{\mu S}$ & $\mathbf{T}_{\mathbf{2}} / \boldsymbol{\mu} \mathbf{S}$ & \multicolumn{2}{c}{$\mathbf{Q}_{\mathrm{Ln}} / \%$} \\
& & & $\lambda_{\mathrm{ex}}=380 \mathrm{~nm}$ & $\lambda_{\mathrm{ex}}=600 \mathrm{~nm}$ \\
\hline \multirow{2}{*}{ MOF-1114(Yb) } & $\mathrm{H}_{2} \mathrm{O}$ & $\begin{array}{c}0.43(1): \\
100 \%\end{array}$ & n.a. & $3.55(5) \cdot 10^{-3}$ & $3.38(7) \cdot 10^{-3}$ \\
& $\begin{array}{c}\mathrm{H}_{2} \mathrm{O}+ \\
\text { Tween } 20\end{array}$ & $\begin{array}{c}0.43(1): \\
100 \%\end{array}$ & n.a. & $4.0(1) \cdot 10^{-3}$ & $4.3(1) \cdot 10^{-3}$
\end{tabular}

Figure S19: (A) $\mathrm{Yb}^{3+}$ emission spectra of miniaturized MOF-1114(Yb) in $\mathrm{H}_{2} \mathrm{O}$ and in $\mathrm{H}_{2} \mathrm{O}$ with $0.5 \%$

Tween-20 under $\lambda_{\mathrm{ex}}=380 \mathrm{~nm}$ and $\lambda_{\mathrm{ex}}=500 \mathrm{~nm}$ excitation; (B) $\mathrm{Yb}^{3+}$ excitation spectra at $\lambda_{\mathrm{em}}=980 \mathrm{~nm}$ in $\mathrm{H}_{2} \mathrm{O}$ and in $\mathrm{H}_{2} \mathrm{O}$ with $0.5 \%$ Tween-20; (C) Luminescence lifetimes $(\tau)$, measured under $355 \mathrm{~nm}$ excitation, and quantum yields $\left(\mathrm{Q}_{\mathrm{Ln}}\right) .2 \sigma$ values are given between parentheses. Measurements taken with crystals submerged in DMF. 


\section{Cell Culture:}

RAW 264.7 cells (a macrophage cell line, ATCC, TIB-71) were cultured in DMEM medium high glucose (Sigma) supplemented with 10\% (vol:vol) fetal bovine serum (FBS, Sigma), 100 $\mathrm{U} / \mathrm{mL}$ penicillin (Sigma), and $100 \mu \mathrm{g} / \mathrm{mL}$ streptomycin (Sigma). Cells were routinely cultured at $37{ }^{\circ} \mathrm{C}$ in a humidified incubator in a $95 \%$ air $/ 5 \% \mathrm{CO}_{2}$ atm and passaged by detaching cells with a cell scraper.

RAW 264.7 cells were seeded in a 8 -chamber Lab-Tek (1.0 borosilicate coverglass system) at 50000 cells/chamber in a final volume of $400 \mu \mathrm{L}$ of complete medium.

After $48 \mathrm{~h}$, cell culture medium was removed and MOFs to be tested were added. The miniaturized MOF in water with $0.5 \%$ Tween-20 was diluted 100 times in complete medium before addition to cells, in order to not exceed $0.005 \%$ as final concentration of Tween-20 and to not induce cell toxicity. ${ }^{5}$ The miniaturized MOF was incubated for $18 \mathrm{~h}$ with RAW 264.7 cells.

Prior to microscopy imaging, RAW 264.7 cells were washed 3 times with $500 \mu \mathrm{L}$ OptiMEM without phenol red (Gibco) supplemented with 2\% (vol:vol) fetal bovine serum (FBS, Sigma), $100 \mathrm{U} / \mathrm{mL}$ penicillin (Sigma), and $100 \mu \mathrm{g} / \mathrm{mL}$ streptomycin (Sigma). RAW 264.7 cells were kept in $200 \mu \mathrm{L}$ of OptiMEM for microscopy imaging. 


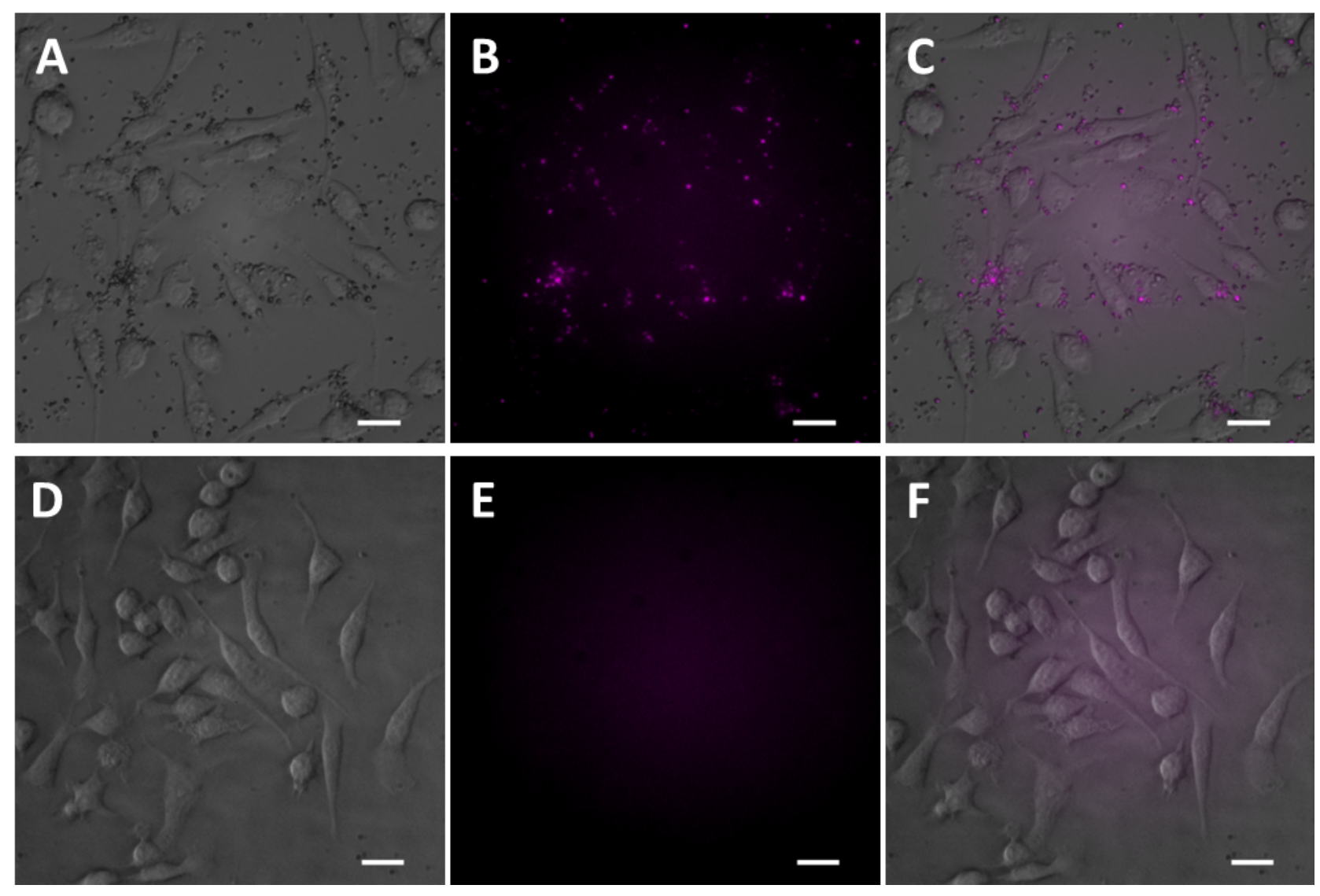

Figure S20: Microscopy images of RAW 264.7 cells incubated with miniaturized MOF in water with $0.5 \%$ Tween-20 (A, B, C) and RAW 264.7 control cells (D, E, F). Brightfield imaging (A, D), NIR epifluorescence imaging (B, E), and merge (C, F). $\lambda_{\text {ex }}: 482 \mathrm{~nm}$ band pass $35 \mathrm{~nm}, \lambda_{\mathrm{em}}: 785 \mathrm{~nm}$ long pass, $\tau_{\text {exp: }}$ : 2 s. Objective $40 \times$. Scale bar $=20 \mu \mathrm{m}$. 


\section{References}

1. Luo, T.-Y.; Liu, C.; Eliseeva, S. V.; Muldoon, P. F.; Petoud, S.; Rosi, N. L., Rare Earth pcu Metal-Organic Framework Platform Based on $\mathrm{RE}_{4}\left(\mu_{3}-\mathrm{OH}\right)_{4}(\mathrm{COO})_{6}{ }^{2+}$ Clusters: Rational Design, Directed Synthesis, and Deliberate Tuning of Excitation Wavelengths. $J$. Am.Chem. Soc., 2017, 139, 9333.

2. Dolomanov, O. V.; Bourhis, L. J.; Gildea, R. J.; Howard, J. A. K.; Puschmann, H., OLEX2: a complete structure solution, refinement and analysis program. J. Appl. Crystallogr. 2009, 42, 339.

3. Sheldrick, G., SHELXT - Integrated space-group and crystal-structure determination. Acta. Cryst. 2015, A71, 3.

4. Sheldrick, G., A short history of SHELX. Acta. Cryst. 2008, A64, 112.

5. Yang, S.; Liu, J.; Chen, Y.; Jiang, J., Reversal effect of Tween-20 on multidrug resistance in tumor cells in vitro. Biomed. Pharmacother. 2012, 66, 187. 